\title{
SPOTS: The Search for Planets Orbiting Two Stars
}

\section{First constraints on the frequency of sub-stellar companions on wide circumbinary orbits}

\author{
M. Bonavita ${ }^{1,2}$, S. Desidera ${ }^{2}$, C. Thalmann ${ }^{3}$, M. Janson ${ }^{4}$, A. Vigan ${ }^{5}$, G. Chauvin ${ }^{6}$, and J. Lannier ${ }^{6}$ \\ ${ }^{1}$ Institute for Astronomy, The University of Edinburgh, Royal Observatory, Blackford Hill, Edinburgh, EH9 3HJ, UK \\ e-mail: mbonav@roe.ac.uk \\ 2 INAF-Osservatorio Astronomico di Padova, Vicolo dell'Osservatorio 5, 35122 Padova, Italy \\ 3 Institute for Astronomy, ETH Zurich, Wolfgang-Pauli Strasse 27, 8093 Zurich, Switzerland \\ 4 Department of Astronomy, Stockholm University, AlbaNova University Center, 10691 Stockholm, Sweden \\ 5 Aix Marseille Université, CNRS, LAM (Laboratoire d'Astrophysique de Marseille) UMR 7326, 13388 Marseille, France \\ ${ }^{6}$ Institut de Planétologie et d'Astrophysique de Grenoble, UJF, CNRS, 414 rue de la piscine, 38400 Saint-Martin-d'Hères, France
}

Received 1 February 2016 / Accepted 12 May 2016

\begin{abstract}
A large number of direct imaging surveys for exoplanets have been performed in recent years, yielding the first directly imaged planets and providing constraints on the prevalence and distribution of wide planetary systems. However, like most of the radial velocity ones, these generally focus on single stars, hence binaries and higher-order multiples have not been studied to the same level of scrutiny. This motivated the Search for Planets Orbiting Two Stars (SPOTS) survey, which is an ongoing direct imaging study of a large sample of close binaries, started with VLT/NACO and now continuing with VLT/SPHERE. To complement this survey, we have identified the close binary targets in 24 published direct imaging surveys. Here we present our statistical analysis of this combined body of data. We analysed a sample of 117 tight binary systems, using a combined Monte Carlo and Bayesian approach to derive the expected values of the frequency of companions, for different values of the companion's semi-major axis. Our analysis suggest that the frequency of sub-stellar companions in wide orbit is moderately low ( $\$ 13 \%$ with a best value of $6 \%$ at $95 \%$ confidence level) and not significantly different between single stars and tight binaries. One implication of this result is that the very high frequency of circumbinary planets in wide orbits around post-common envelope binaries, implied by eclipse timing, cannot be uniquely due to planets formed before the common-envelope phase (first generation planets), supporting instead the second generation planet formation or a non-Keplerian origin of the timing variations.
\end{abstract}

Key words. binaries: visual - binaries: spectroscopic

\section{Introduction}

In the past decade, an increasing amount of effort has been spent on studying the formation and evolution of planets in the environment of binary host star systems (see e.g. the book "Planets in Binaries", Haghighipour 2010). More than one hundred planets have been found in binary systems to date ${ }^{1}$. Most of these discoveries have been made with indirect detection methods such as Doppler spectroscopy or transit photometry methods, which are heavily biased towards planets with short orbital periods and, therefore, favour circumstellar ("s-type") configurations around individual components of wide binary systems. Despite this bias, about 20 of these planets have been found in circumbinary ("p-type") orbits encompassing tight binary systems, hinting at the existence of an extensive unseen population of circumbinary planets.

Direct imaging, on the other hand, is a powerful planet detection technique particularly well suited to planets on wide orbits, which complements the limited parameter space of the indirect detection methods. A number of direct imaging

\footnotetext{
1 exoplanets.org database (Wright et al. 2011),
} www . exoplanets. eu (Schneider et al. 2011). surveys have been published to date (e.g., Lafrenière et al. 2007; Vigan et al. 2012; Janson et al. 2013a; Biller et al. 2013; Rameau et al. 2013b; Daemgen et al. 2015), which have resulted in the discovery of several planets (e.g. Marois et al. 2010; Lagrange et al. 2010; Kuzuhara et al. 2013; Carson et al. 2013; Rameau et al. 2013a) and brown-dwarf companions (e.g. Thalmann et al. 2009; Biller et al. 2010; Bonavita et al. 2014). Such surveys typically reject binary systems from their target sample. Although many previously unknown tight systems were still included in their target lists, the population of wide-orbit planets in such systems still remains largely unexplored.

To address this, the Search for Planets Orbiting Two Stars project (SPOTS; Thalmann et al. 2014, hereafter Paper I) is conducting the first dedicated direct imaging survey for circumbinary planets. Our long-term goal is to observe a large sample of young nearby tight binary systems with the VLT NaCo, VLT SPHERE, and LBT/LMIRCAM facilities. The NaCo-based first stage of the survey, which comprises 27 targets, completed its exploratory observations in 2013 (Paper I) and the follow-up observations to confirm the physical association of planet candidates is in progress. Additional close binary targets are being observed with the newly installed direct imaging instrument 
SPHERE (Beuzit et al. 2010) and with LMIRCAM at LBT in the context of the LEECH project (Skemer et al. 2014), increasing the sensitivity to planetary companions at close separation. Although the survey is not yet completed, it has already yielded a first discovery: the sharp highly asymmetric features in the circumbinary protoplanetary disk around Ak Sco imaged with SPHERE (Janson et al. 2016).

A discussion of the survey's scientific background, observational strategy, and first results is presented in Paper I. The scientific justification can be summarised in the following four main points:

- Theoretical and observational evidence suggests that circumbinary planets constitute a significant fraction of the overall planet population, and therefore merit exploration.

- With appropriate target selection, the host binarity has no detrimental effects on observation and data reduction. The detectability of planets around a tight binary may in fact be superior to that around a single star of equal system brightness, since the greater total system mass is expected to correlate with a greater amount of planet-forming material.

- Dynamic interactions with the host binary can launch circumbinary planets that formed or migrated close to the system centre onto wide orbits, where they are more easily imaged.

- Measuring differences in the planet demographics between circumbinary and single-star target samples may bring new insights into the physics of planet formation and evolution that would be inaccessible to surveys of single stars only.

Details and references for these claims are listed in Thalmann et al. (2014).

Here, we present a statistical analysis of the combined body of existing high-contrast imaging constraints on circumbinary planets to complement our ongoing survey. Indeed, while several of the available surveys intended to avoid binaries, or at least close visual binaries, the census of stellar multiplicity was highly incomplete at the time of the execution of the observations. The direct imaging surveys provided themselves the best census of close visual binaries, with each survey contributing typically with several new discoveries.

For this purpose, we searched the target lists of 23 published direct imaging surveys, looking for tight binaries, collected their contrast curves, and compared them to synthetic circumbinary planet populations using the QMESS code (Bonavita et al. 2013). The target sample is presented in Sect. 2, the stellar and binary properties in Sect. 3 and the statistical analysis is described in Sect. 4. Finally the results are summarised and discussed in Sect. 5.

\section{Target samples}

\subsection{The circumbinary sample}

Our initial sample was built merging the target lists of the several recent deep imaging surveys with sensitivity adequate for detection of giant planets. Among these are some of the largest deep imaging surveys performed to date, such as the VLT/NaCo large program (NLP) by Chauvin et al. (2015), the Planets around Low-mass Stars (PALMS) survey (Bowler et al. 2015), the Strategic Exploration of Exoplanets and Disk with Subaru (SEEDS) survey (Brandt et al. 2014a; Janson et al. 2013a, B13 and J13, respectively) and the Gemini NICI Planet-Finding Campaign (Nielsen et al. 2013; Biller et al. 2013, N13 and BN13, respectively). The main characteristics of all the surveys considered in this paper are reported in Table 1 . To these, we added also the low-mass spectroscopic binary CHXR 74, which orbit has been constrained by Joergens et al. (2012, JJ12).

We also included some target from a HST/NICMOS survey of 116 young $(<30 \mathrm{Myr})$ nearby $(<60 \mathrm{pc})$ stars (Song et al. private communication, see also Song et al. 2006). Each target was observed at two spacecraft roll angles in successive HST orbits. After standard cosmetics correction, the two roll angle images were recentered and subtracted to suppress the stellar Light contribution. Additional Fourier filtering was applied to remove point spread function (PSF) low-spatial frequencies to search for faint point-like sources in the star vicinity. Detection limits and maps were derived using a $5 \times 5$ pixels sliding box over the whole image and flux calibrated considering the standard NICMOS photometric calibration in the F160W observing filters ${ }^{2}$.

For all the targets an extensive search for multiplicity was performed in binary catalogues such as the HIPPARCOS and Tycho Catalogues (Perryman \& ESA 1997), the Catalogue of the Components of Double and Multiple Stars (CCDM; Dommanget \& Nys 2002), the Washington Visual Double Star Catalogue (WDS; Worley \& Douglass 1997), the 9th catalogue of spectroscopic orbits (SB9; Pourbaix et al. 2004), the SACY database (Torres et al. 2006), the Geneva-Copenhagen survey (Nordström et al. 2004). We also considered the literature on individual targets as well as from the direct imaging surveys themselves, which resolved for the first time a number of pairs, making the input papers the best sources to be used to identify close visual binaries. Ambiguous cases such as candidate binaries with astrometric accelerations only or with position above sequence of coeval stars in colour-magnitude diagram are not included in our sample of binaries. We also note that several of the targets of imaging surveys are lacking radial velocity monitoring, thus the census of spectroscopic binaries is likely incomplete.

When searching for circumbinary planet hosts in such samples, one must take into account that most of these surveys includes severe selection biases against binary targets. Most surveys in fact excluded known binaries with separations smaller than 2 arcsec. Nevertheless, a significant number of binary and multiple targets are found in this surveys, not being known at the time of the target list compilation, or resolved for the first time during the searches themselves.

Of course, wide binaries are not suited to a search for circumbinary planets. We fixed as a limit for our investigation the systems for which the inner limit of dynamical stability for circumbinary planets (see Sect. 3.2 for definition and determination) is smaller than $50 \mathrm{au}$. This limit roughly corresponds to the expected truncation limit of the circumbinary disk. The adopted limit is significantly larger than the dynamical stability limits for the circumbinary systems discovered by Kepler but it can be considered as conservative when looking at the properties of some binaries hosting well-studied circumbinary disks such as GG Tau A $(a \sim 60$ au, Köhler 2011) and SR24N ( $a \sim 32$ au, Andrews \& Williams 2005).

Therefore, while the adopted limit is somewhat arbitrary, it appears reasonable for the identification of a sample of systems for which the presence of circumbinary planets is possible and worth to be explored.

With such selection criteria, a total of 139 targets were selected. Taking into account the overlap between the various surveys considered, our final sample for the search for circumbinary planets (hereafter CBIN sample) includes 117 unique systems.

\footnotetext{
2 Please refer to: http://www.stsci.edu/hst/nicmos/ performance/photometry
} 
M. Bonavita et al:: Constraints on the frequency of circumbinary planets in wide orbits

Table 1. Characteristics of the surveys considered to build the circumbinary (CBIN) sample.

\begin{tabular}{lcccccl}
\hline \hline Source & Instrument & Technique & Filter & $N_{S r v}^{2}$ & $N_{C B I N}^{3}$ & Reference \\
\hline L05 & HST/NICMOS & COR & $H(1.4-1.8)$ & 45 & 6 & Lowrance et al. (2005) \\
B06 & VLT/NACO & COR & $K_{\mathrm{S}} / H$ & 17 & 3 & Brandeker et al. (2006) \\
B07 & VLT-NACO/MMT & SDI & $H$ & 45 & 7 & Biller et al. (2007) \\
K07 & VLT/NACO & DI & $L$ & 22 & 4 & Kasper et al. (2007) \\
GDPS & GEMINI/NIRI & SDI & $H$ & 85 & 8 & Lafrenière et al. (2007) \\
CH10 & VLT/NACO & COR & $H / K_{\mathrm{S}}$ & 91 & 9 & Chauvin et al. (2010) \\
H10 & Clio/MMT & ADI & $L^{\prime} / M$ & 54 & 3 & Heinze et al. (2010) \\
JB11 & GEMINI/NIRI & ADI & $K_{H} / H$ & 18 & 3 & Janson et al. (2011) \\
JJ12 & VLT/NACO & DI & $K_{\mathrm{S}}$ & 1 & 1 & Joergens et al. (2012) \\
V12 & VLT/NACO, NIRI & ADI & $K_{\mathrm{S}} / \mathrm{H}^{\prime} / \mathrm{CH} 4$ & 42 & 3 & Vigan et al. (2012) \\
R13 & VLT/NACO & ADI & $L^{\prime}$ & 59 & 3 & Rameau et al. (2013b) \\
B13 & SUBARU/HiCiao & DI/ADI/PDI & $\mathrm{H}$ & 63 & 6 & Brandt et al. (2014a) \\
J13 & SUBARU/HiCiao & ADI & $H$ & 50 & 4 & Janson et al. (2013a) \\
Y13 & SUBARU/HiCiao & ADI & $H / K_{\mathrm{S}}$ & 20 & 3 & Yamamoto et al. (2013) \\
N13 & GEMINI/NICI & ADI/ASDI & $H$ & 70 & 4 & Nielsen et al. (2013) \\
BN13 & GEMINI/NICI & ADI/ASDI & $H$ & 80 & 4 & Biller et al. (2013) \\
JL13 & GEMINI/NICI & DI/ADI & $K_{\mathrm{S}}$ & 138 & 5 & Janson et al. (2013b) \\
L14 & GEMINI/NIRI & DI/ADI & $K_{\mathrm{S}}$ & 91 & 18 & Lafrenière et al. (2014) \\
SONG & HST & ADI & $H$ & 116 & 14 & Song et al., priv. comm. \\
M14 & VLT/NACO & ASDI & $H$ & 16 & 1 & Maire et al. (2014) \\
NLP & VLT/NACO & DI/ADI & $H$ & 110 & 8 & Chauvin et al. (2015) \\
D15 & GEMINI/NIRI & DI & $K_{\mathrm{S}}$ & 64 & 4 & Daemgen et al. (2015) \\
B15 & SUBARU/HiCiAO & DI/ADI & $K_{\mathrm{S}}$ & 31 & 5 & Bowler et al. (2015) \\
& KECK/NIRC2/N & DI/ADI & $H$ & 59 & 3 & \\
L15 & VLT/NACO & ADI & $L^{\prime}$ & 58 & 10 & Lannier et al. (2016) \\
\hline
\end{tabular}

Notes. ${ }^{(1)}$ Techniques: $\mathbf{C O R}=$ Coronagraphy; SDI = Spectral Differential Imaging; DI = Direct Imaging; ADI = Angular Differential Imaging; PDI = Polarized Differential Imaging; ASDI = Angular and Spectral Differential Imaging. ${ }^{(2)}$ Total number of targets included in the original survey. ${ }^{(3)}$ Number of stars considered in our study.

The stellar and binary parameters of the stars in the CBIN sample are derived following the prescriptions described in Sect. 3 and are listed in Table B.1.

It is interesting for the purposes of our statistical analysis and for comparison with other results (e.g., from Kepler space mission) to obtain an ensemble view of the properties of the sample. To this aim, Fig. 1 shows histograms and plots of several relevant parameters, derived as described in Sect. 3.1. As expected, the sample is dominated by young stars, with median age $\sim 50 \mathrm{Myr}$. Nevertheless, several old stars are present, mostly tidally-locked binaries originally classified as young due to their high activity levels. The median distance of the systems is $45 \mathrm{pc}$, with a significant number of objects (25\%) at distances larger than $100 \mathrm{pc}$, mostly members of Sco-Cen groups. The total system mass lies between 0.22 to $20.8 M_{\odot}$, with a median value of $1.34 M_{\odot}$. The distribution of critical semi-major axis has a median value of $10 \mathrm{au}$, with $48 \%$ of systems with $a_{\text {crit }}<10$ au. Binaries at larger $a_{\text {crit }}$ are under-represented in the sample with respect to unbiased samples due to the exclusion of previously known close visual binaries in most of the imaging surveys. The mass ratio distribution is fairly uniform, with a median value of 0.61 .

\subsection{The control sample}

In order to ensure a consistent comparison of our results with those obtained for single stars, we carried an independent analysis of the sample described by Brandt et al. (2014b). All the binaries used for our analysis were removed from the sample, together with those targets for which the detection limits were not available. We also removed from the comparison sample the stars with stellar companions within 100 au. As suggested by Bonavita \& Desidera (2007) and, more recently by Duchêne (2010), systems with separation $>100$ au are in fact indistinguishable from single stars as far as the initial conditions and end product of planet formation are concerned. With these assumptions, the final control sample (hereafter SS sample) includes 205 stars.

\section{CBIN sample properties}

The CBIN sample is quite heterogeneous in terms of stellar and binary properties, as expected considering the original selection criteria in the parent surveys, which are focused in some cases of specific types of stars (low mass stars, early type stars, specific young moving groups), the presence or not of biases against specific types of binaries, etc. In this section, we present our determination of stellar and binary parameters for the systems included in our sample.

\subsection{Stellar parameters}

\subsubsection{Stellar Ages}

Even if their evolution is not completely understood (see Fortney et al. 2008), giant planets are in fact thought to be more luminous at young ages, their luminosity fading with time, as they cool down (see Baraffe et al. 2003; Marley et al. 2007). Thus, observing younger targets increases the probability to find 

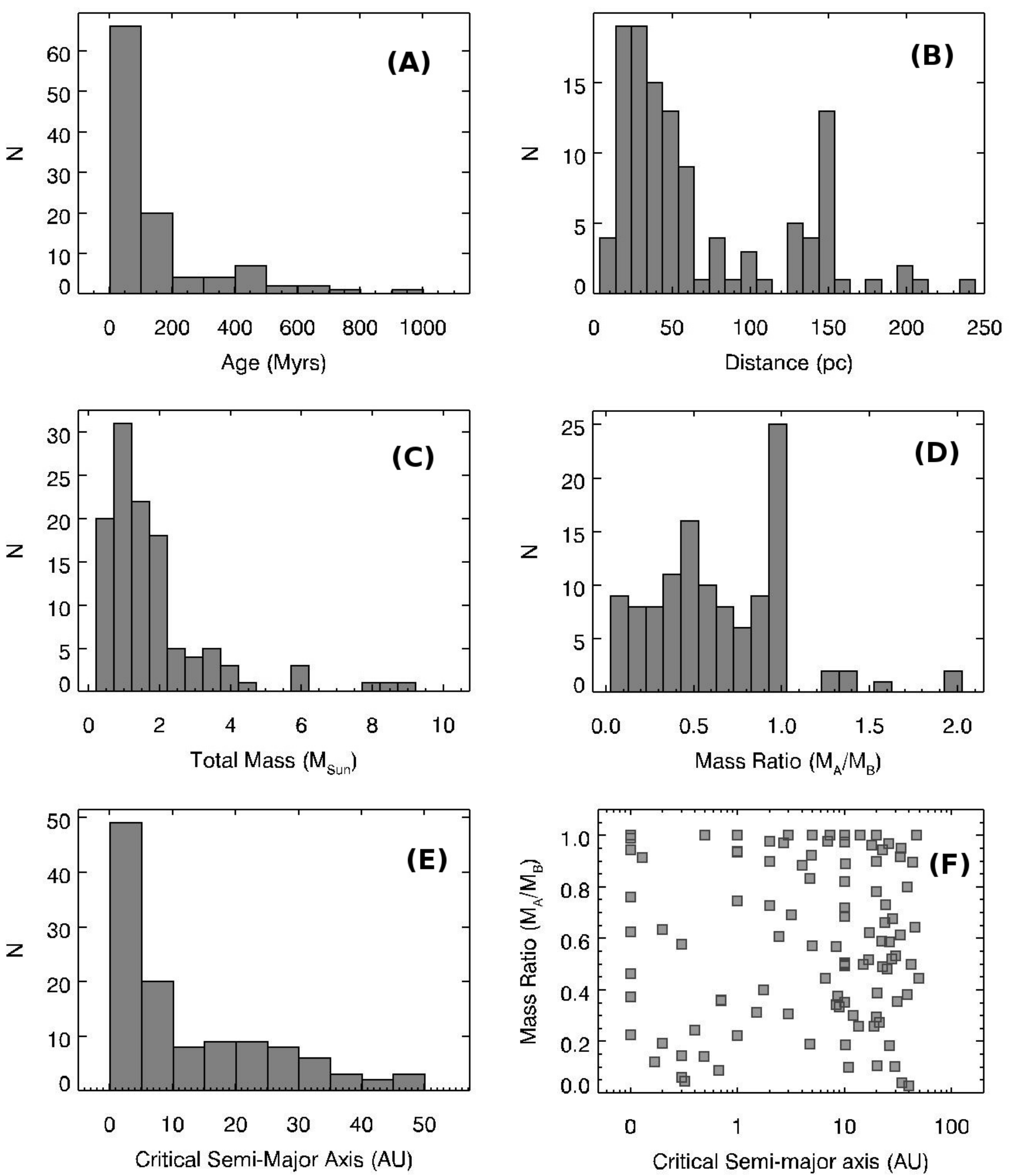

Fig. 1. Properties of the stars in the CBIN sample. A) histogram of stellar ages; B) histogram of stellar distances; C) histogram of total system masses; D) histogram of the system mass-ratio; E) histogram of critical semi-major axis for dynamical stability of planetary companions $\left(a_{\text {crit }}\right)$; F) inner limit for circumbinary planet stability $\left(a_{\text {crit }}\right)$ vs. binary mass ratio.

smaller companions by raising the planet/mass contrast, espe- lists for the surveys we considered were assembled on the basis cially in the IR domain. Therefore, most of the original target of the young ages. 
In the past few years, significant efforts were devoted to the identification of nearby young stars and to the determination of their basic parameters. However, the determination of stellar age is still a challenging task (Soderblom et al. 2014) and stellar multiplicity represent an additional source of complications due to blending of the spectral features and lack of spatially resolved fluxes for most of the systems studied in the present paper. Furthermore, in very close binaries the components are tidally locked and so they have a short rotation period, thus mimicking some of the characteristics of young stars, such as high levels of chromospheric and coronal activity. There are also claims that Lithium abundance, another widely used age indicator, is altered in tidally-locked binaries (Pallavicini et al. 1992b).

There are several cases of stars included in the direct imaging surveys being classified as young thanks to their high level or chromospheric and coronal activity but the subsequent identification of their nature as close spectroscopic binaries suggest that these are due to tidal locking and not to young age. In these cases, the determination of the stellar ages is very critical, especially when the lack of orbital solution prevents the study of the system kinematic. In some cases, we conservatively adopt an age of $4 \mathrm{Gyr}$, given the lack of specific constraints on stellar age. In some other cases, multiplicity was not known or in any case not taken into account in the derivation of stellar properties, resulting in biased parameters (e.g., photometric distances and then kinematic parameters).

In general, we followed the procedures described in Desidera et al. (2015) to derive stellar ages. For field stars, stellar ages were obtained from a variety of age indicators (lithium, chromospheric emission, coronal emission, rotation period, kinematic, isochrone fitting), exploiting measurements and age calibrations published after the original papers presenting the direct imaging surveys. For this reason, in several cases the system ages adopted in this work differ from those of the original papers. For close binary systems evolved through mass exchanges phase, ages and individual masses were taken from papers dedicated to the study of these objects.

Age is easier to determine in young associations, because a variety of stellar dating techniques can be used for stars of different masses (stellar models for low-mass stars and massive evolved stars, lithium, etc.,) or for the association as a whole (kinematic age derived from relative velocities and position of the members).

The membership of the targets to various young associations and clusters was taken from several literature sources (Zuckerman \& Song 2004; Torres et al. 2008; Zuckerman et al. 2011; Malo et al. 2013) and on studies of individual objects. Following the most recent results published in the literature in the last year, the ages of several young moving groups were revised with respect to those adopted in Desidera et al. (2015) and in Paper I. For $\beta$ Pic, Tuc-Hor, Columba, AB Dor, TW Hya associations and $\eta$ Cha open cluster we adopt the ages from Bell et al. (2015). For Argus-IC 2391, we adopt the Li-depletion boundary age by Barrado y Navascués et al. (2004), considering the ambiguities in the isochrone fitting discussed in Bell et al. (2015). For Sco-Cen groups, we adopt the ages from Pecaut et al. (2012), as already done in Desidera et al. (2015). They are based on the same technique employed in Bell et al. (2015), even if there are differences in some details of the isochrone fitting procedure. The resulting age ranking is also consistent with the result that the Lower Crux Centaurus group (LCC) is younger than $\beta$ Pic moving group (MG) members, as found by Song et al. (2012) from $\mathrm{Li} \mathrm{EW}$. To be consistent with the upward revision of ages of most moving groups, we also revise the age of the Carina-Near moving group to $250 \mathrm{Myr}$. This is consistent with the recent gyro-chronology age of the nearly coeval Her-Lyr association Eisenbeiss et al. (2013), although we do not have targets from this last group in our list. For Pleiades and Hyades open clusters we adopt 125 and $625 \mathrm{Myr}$, respectively. For Castor and Ursa Major moving groups we adopt 320 and 500 Myr, respectively.

Details of the age indicators and membership to groups for individual targets are provided in Appendix A, The ages of moving groups as described above were also adopted for the members included in the comparison sample of single stars considered in the statistical analysis in Sect. 4.4

\subsubsection{Stellar distances}

Trigonometric distance from HIPPARcos New Reduction (Van Leeuwen 2007) or other individual sources were adopted when available. For other members of groups Torres et al. (2008) photometric+kinematic distances were adopted. For members of Upper-Scorpius without trigonometric parallax, a distance of $145 \mathrm{pc}$ is adopted. For field stars without trigonometric parallax, photometric distances were derived using empirical sequences for different ages determined from members of moving groups, as described in Desidera et al. (2015).

\subsubsection{Stellar masses}

Stellar masses were derived in most cases through stellar models for the adopted ages. In some case individual dynamical masses or mass ratio are available from orbital solution and we took into account this information. For the spectroscopic binaries for which only minimum mass of the companion is available from the orbital solution, we adopt this value to derive the critical semi-major axis for dynamical stability (see Sect. 3.2). For the spectroscopic binaries for which minimum mass is not available (e.g. only indication for short period RV variations without orbital solution), we adopt a mass equal to half of that of the primary for the computation of the dynamical stability limit. Figs. 1C and 1D show the histograms of the total mass $\left(M_{A}+M_{B}\right)$ and of the mass ratio $\left(q=M_{B} / M_{A}\right)$, respectively, for the systems in the CBIN sample. Note that for the few systems where the secondary is a tight pair (see Sect. 3.2.1 and Table 2 for details) the total mass of the two components was considered, thus resulting in a value of $q>1$.

\subsection{Binary parameters}

The properties of the systems included in the CBIN sample are listed in Table B.1. References and details on individual systems are provided in Appendix A. When the complete orbital solution is known, semi-major axis and eccentricity are listed. For systems for which no reliable semi major axis was available, we made the estimation that $a(\mathrm{au}) \sim \rho(\operatorname{arcsec}) d(\mathrm{pc})$. This relies on the assumption of a flat eccentricity distribution, based on the results of Raghavan et al. (2010).

For spectroscopic binaries the masses as described in Sect. 3.1.3 were adopted.

Table B.1 also reports the values of the critical semi-major axis for dynamical stability $\left(a_{\text {crit }}\right)$, calculated following the approach of Holman \& Wiegert (1999), For the circumbinary case 
Table 2. Additional wide companion around the close pairs in the CBIN sample.

\begin{tabular}{|c|c|c|c|c|c|c|c|c|}
\hline$\#^{1}$ & Star ID & $\begin{array}{c}M_{\text {Target }}^{2} \\
M_{\odot}\end{array}$ & $\begin{array}{c}M_{\text {Outer }}^{3} \\
M_{\odot}\end{array}$ & $\begin{array}{c}\rho \\
\left({ }^{\prime \prime}\right)\end{array}$ & $\begin{array}{c}a \\
(\mathrm{au})\end{array}$ & $e$ & $\begin{array}{l}a_{C S}^{4} \\
(\mathrm{au})\end{array}$ & Notes \\
\hline 6 & HIP 4967 & 0.88 & 1.22 & 25.6 & 765 & - & 81 & \\
\hline 10 & HIP 12413 & 2.63 & 0.40 & 23.8 & 947 & - & 159 & \\
\hline 12 & HIP 12638 & 1.19 & 0.80 & 14.57 & 662 & - & 87 & \\
\hline 13 & HIP 13081 & 1.16 & 0.16 & 20.0 & 493 & - & 83 & \\
\hline 23 & RX J0415.8+3100 & 1.16 & 0.62 & 0.95 & 190 & - & 26 & \\
\hline 25 & HIP 21482 & 1.03 & 0.67 & 126 & 2268 & - & 300 & \\
\hline 26 & GJ 3305 & 1.35 & 1.60 & 66.0 & 1942 & - & 217 & \\
\hline 29 & HIP 23296 & 1.79 & 0.09 & 9.17 & 455 & - & 82 & \\
\hline 30 & HIP 23418 & 0.41 & 0.25 & 1.37 & 34 & - & 4 & \\
\hline 33 & AB Dor AC & 0.96 & 0.32 & 9.0 & 136 & - & 21 & \\
\hline 34 & AB Dor Bab & 0.32 & 0.96 & 9.0 & 136 & - & 11 & \\
\hline 38 & HIP 35564 & 2.19 & 2.40 & 9.0 & 285 & - & 32 & quintuple system \\
\hline 41 & GJ $278 \mathrm{C}$ & 1.20 & 4.83 & 72 & 1073 & - & 82 & Castor, sextuple system \\
\hline 43 & HIP 39896 A & 1.00 & 0.72 & 14 & 298 & - & 38 & close pair of M dwarfs \\
\hline 44 & HIP 39896 B & 0.72 & 1.00 & 14 & 298 & - & 32 & quadruple \\
\hline 52 & HIP 49669 & 3.70 & 1.10 & 175 & 4165 & - & 644 & quadruple \\
\hline 59 & HD 102982 & 2.18 & 0.33 & 0.90 & 56 & - & 9 & \\
\hline 71 & HIP 72399 & 1.12 & 0.71 & 11.0 & 507 & - & 67 & \\
\hline 75 & HIP 76629 & 1.23 & 0.4 & 10.2 & 393 & - & 60 & \\
\hline 83 & 1RXS J160210.1-2241.28 & 1.35 & 0.53 & 0.300 & 43 & - & 6 & \\
\hline 90 & HIP 79097 & 3.06 & 0.75 & 0.814 & 163 & - & 26 & \\
\hline 93 & HIP 79643 B & 1.05 & 2.10 & 1.24 & 262 & - & 25 & \\
\hline 95 & HIP 84586 & 2.05 & 0.25 & 33 & 1038 & - & 178 & \\
\hline 97 & HIP 86346 & 1.23 & 0.30 & 19.6 & 590 & - & 94 & \\
\hline 99 & CD-64 $1208 \mathrm{~A}$ & 1.31 & 1.60 & 70 & 1998 & & 222 & \\
\hline 102 & HIP 94863 & 1.46 & 0.26 & 9.4 & 394 & - & 65 & \\
\hline 104 & HIP 97255 & $\sim 1.40$ & 0.60 & 9.90 & 307 & - & 44 & \\
\hline 105 & 2MASSJ19560294-3207186 & 0.30 & 0.55 & 26.0 & 1430 & - & 140 & \\
\hline 110 & HIP 105441 & 1.27 & 0.65 & 26.1 & 787 & - & 110 & \\
\hline 113 & HIP 108195 & 3.0 & 0.2 & 4.89 & 227 & - & 40 & \\
\hline 116 & PMM 366328 AB & 1.82 & 0.56 & 24.0 & 1440 & - & 222 & \\
\hline
\end{tabular}

Notes. ${ }^{(1)}$ Reference number from Table B.1. ${ }^{(2)}$ Mass of the inner pair $\left(M_{A}+M_{B}\right.$ from Table 2). ${ }^{(3)}$ Mass of the additional companion. ${ }^{(4)}$ Outer limit for the stability, calculated using Eq. (2).

this inner limit for the stability is given by:

$$
\begin{aligned}
a_{\text {crit }}=a_{C B}= & \left(1.60+4.12 \mu+5.10 e_{b}\right) a_{b} \\
& +\left(-4.27 \mu e_{b}-5.09 \mu^{2}\right) a_{b} \\
& +\left(-2.22 e_{b}^{2}+4.61 e_{b}^{2} \mu^{2}\right) a_{b} .
\end{aligned}
$$

In the equation we assume $\mu=\frac{M_{B}}{M_{A}+M_{B}}$, where $M_{A}$ is the mass of the primary star, $M_{B}$ the mass of the secondary and $a_{\text {bin }}$ and $e_{\text {bin }}$ are the semi major axis and the eccentricity of the binary orbit. In agreement with the assumption used for the semi-major axis calculation, an eccentricity value of 0.5 was adopted for the systems for which no information on the orbit was available.

We choose $a_{\text {crit }}$ as a reference value because it is a physical quantity that better represents the dynamical effects due to a companion on planet formation and stability, including both the orbital parameters and mass ratio. Only planets outside the $a_{\text {crit }}$ limit for circumbinary planets were considered in the statistical analysis.

\subsubsection{Higher order systems}

There are several cases among our targets showing higher order multiplicity. Five systems (Algol, TWA5, BS Ind, V815 Her and HIP 78977) are tight triple systems with an inner pair with period shorter than 5 days and an external component with semimajor axis smaller than 3 au. In these cases, the direct imaging data would be able to detect planets around the three components. The critical semi-major axis for circumbinary planets was derived in these cases considering the sum of the masses of the inner pair, the mass of the outer component and the outer orbital parameters.

There are also several cases of hierarchical systems with an additional component at wide separation (Table 2). In these cases, we considered the dynamical effects on possible circumbinary planets considering the tight binary as a single star with a mass resulting from the sum of the individual components. The limit for the presence of circumbinary planets due the outer companion(s) is therefore derived using the equation by Holman \& Wiegert (1999) for circumstellar planets:

$$
\begin{aligned}
a_{\text {crit }}=a_{C S}= & \left(0.464-0.38 \mu+0.361 e_{b}\right) a_{b} \\
& +\left(0.586 \mu e_{b}+0.150 e_{b}^{2}\right) a_{b} \\
& +\left(-0.198 \mu e_{b}^{2}\right) a_{b} .
\end{aligned}
$$

For the 31 systems listed in Table 2 this outer stability limit is smaller than the maximum value considered for the planetary 
semi-major axis (1000 au). Therefore for these targets both the inner and outer limit for the stability have been considered for the statistical analysis (Sect. 4.3).

The few cases of compact triple systems for which the stability limit due to the presence of the outer component is smaller than the limit for circumbinary planets around the central pair were removed from the sample.

\section{Statistical analysis}

\subsection{Statistical formalism}

For our statistical analysis we used a Bayesian approach described in Lafrenière et al. (2007) and in a similar way to what has been done by Vigan et al. (2012) and Brandt et al. (2014b).

Our goal is to link the fraction $f$ of the $N$ systems in our sample hosting at least one companion of mass and semi-major axis in the interval $\left[m_{\min }, m_{\max }\right] \cap\left[a_{\min }, a_{\max }\right]$ with the probability $p$ that such companion would be detected from our observations.

The likelihood of the data given $f$ is

$L\left(\left\{d_{j}\right\} \mid f\right)=\prod_{j=1}^{N}\left(1-f p_{j}\right)^{1-d_{j}} \cdot\left(f p_{j}\right)^{d_{j}}$

where $\left(f p_{j}\right)$ is the probability of detecting a companion around the jth star, $\left(1-f p_{j}\right)$ is the probability of non detection and $\left\{d_{j}\right\}$ denotes the detections made by the observations, such that $d_{j}$ equals 1 if at least one companion is detected around star $j$ and 0 otherwise.

As we have no a priori knowledge of the wide-orbit massive planet frequency, we adopt a maximum ignorance prior, $p(f)=1$. From this prior and the likelihood defined as in Eq. (3) we can use Bayes' theorem to obtain the probability that the fraction of stars having at least one companion is $f$, given our observations $\left\{d_{j}\right\}$, or posterior distribution:

$p\left(f \mid\left\{d_{j}\right\}\right)=\frac{L\left(\left\{d_{j}\right\} \mid f\right) \cdot p(f)}{\int_{0}^{1} L\left(\left\{d_{j}\right\} \mid f\right) \cdot p(f) \mathrm{d} f}$.

For a given confidence level $C L=\alpha$ we can then use this posterior distribution $p\left(f \mid\left\{d_{j}\right\}\right)$ to determine a confidence interval (CI) for $f$ as follows:

$\alpha=\int_{f_{\min }}^{f_{\max }} p\left(f \mid\left\{d_{j}\right\}\right) \mathrm{d} f$

the boundaries of this $\mathrm{CI}$ being the minimal $\left(f_{\min }\right)$ and maximal $\left(f_{\max }\right)$ values of $f$ compatible with our observations.

In case of a null result, clearly $f_{\min }=0$ and the only result of the such analysis would be a constraint on $f_{\max }$.

For a case, like ours, where there are some detections, an equal-tail CI can be assumed, and for a given value of $\alpha, f_{\min }$ and $f_{\max }$ can be obtained by numerically solving the following equations (see Lafrenière et al. 2007):

$$
\begin{aligned}
& \frac{1-\alpha}{2}=\int_{f_{\max }}^{1} p\left(f \mid\left\{d_{j}\right\}\right) \mathrm{d} f \\
& \frac{1-\alpha}{2}=\int_{0}^{f_{\min }} p\left(f \mid\left\{d_{j}\right\}\right) \mathrm{d} f .
\end{aligned}
$$

\subsection{Detection limits}

For each of the targets in the CBIN sample, we collected the available information on the sensitivity in terms of star/planet contrast at a given angular distance from the star. Such detection limits were therefore used to define the discovery space of our search. Even if with many common points, the methods used for the evaluations of the limits are slightly different in the various surveys listed in Table 1, the main discriminant being the way in which the noise estimation is made.

Except for Lowrance et al. (2005), which uses a completely different approach, a Gaussian distribution is assumed for the noise, and a $5-6 \sigma$ level is set for the detection. This is particularly appropriate in case of the ADI data, since the LOCI processing leads to residuals whose distribution closely resembles a Gaussian (see e.g. Lafrenière et al. 2007).

Biller et al. (2013) report 95\% completeness levels rather than $5 \sigma$ thresholds. We therefore used the method described by Brandt et al. (2014b) to convert them into a common framework with the values from the other studies.

In the case of the SONG HST survey, 2D detection maps were used.

The COND models (Allard et al. 2001; Baraffe et al. 2003) were used to convert the sensitivity curves into minimum mass limits for all the stars in the CBIN sample.

\subsection{Detection probability}

In order to evaluate the detection probability $\left(f p_{j}\right)$ for the targets in our sample, we used the QMESS code (Bonavita et al. 2013). The code uses the information on the target stars, together with the detection limits described in Sect. 4.2 to evaluate the probability of detection of companions with semi-major axis up to $1000 \mathrm{au}$ and masses up to $75 M_{\text {Jup }}$. These values were chosen after a series of tests, aimed at constraining the best possible parameter space for our analysis, given the way our sample was constructed.

A dedicated version of the QMESS code was used for the target from the SONG HST survey, as 2D contrast maps were provided instead of 1D contrast curves for this purpose (see Bonavita et al. 2012, for details).

In case several limits were available for the same star, separate runs were performed using each limit singularly. Then the final detection probability map was built by considering, for each grid point, the highest value among the full set. This is equivalent to assume that a planet is detected if it is so in at least one of the images.

The same kind of analysis was repeated for the targets in the control SS sample described in Sect. 2.2.

Figure 2 shows the average detection probability map obtained considering all the stars in both the CBIN sample (left panel) and SS control sample (right panel).

\subsection{Derived companion frequency}

Five of the 117 systems in the CBIN sample have reported detection of additional sub-stellar companions, two of which (HIP 59960b and 2MASS J01033563-5515561 AB b) below the deuterium burning limit. The SS control sample described in Sect. 2.2 includes 7 targets with confirmed sub-stellar companions, including the planetary-mass companions of $\kappa$ And and AB Pic. The sub-stellar companions HN Peg B (Luhman et al. 2007) and MN UMa B (Kirkpatrick et al. 2001) are not included in the statistical analysis being at larger projected separation than 

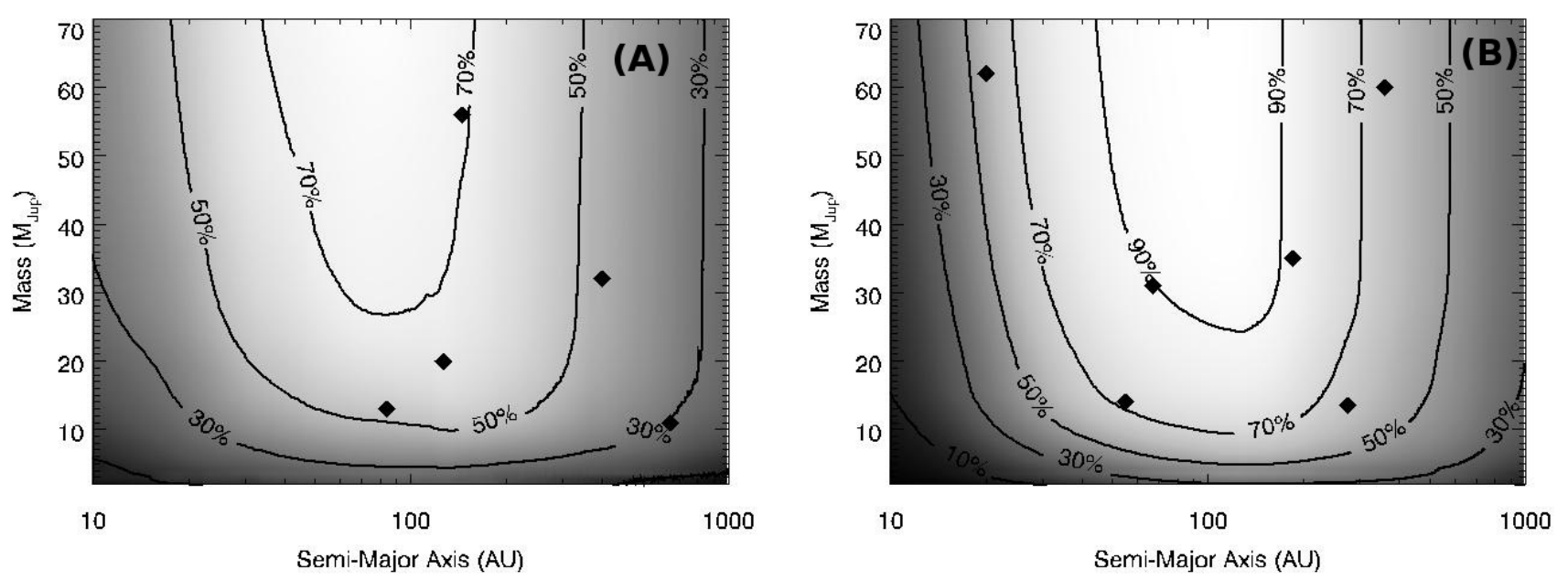

Fig. 2. Average detection probability as a function of planetary mass and semi-major axis. A) Circumbinary (CBIN) Sample; B) comparison (SS) Sample. In both panels the sub-stellar companions reported in Table 3 are marked with filled diamonds.

Table 3. Sub-stellar companions detections.

\begin{tabular}{llccll}
\hline \hline CBIN sample & & & & \\
\hline$\#^{1}$ & ID & Mass $\left(M_{\text {Jup }}\right)$ & Sep (au) & Survey $^{2}$ & Reference $^{3}$ \\
\hline 61 & HIP 59960 b & $11 \pm 2$ & $654 \pm 3$ & JL13 & Bailey et al. (2014) \\
5 & 2MASS J01033563-5515561 AB b & $13 \pm 1$ & 84 & L15 & Delorme et al. (2013) \\
58 & TWA 5 B & 20 & 127 & L05 & Lowrance et al. (1999) \\
22 & HIP 19176 B & 32 & 400 & D15 & Bonavita et al. (2014) \\
20 & H II 1348 B & $56 \pm 3$ & $145 \pm 2.3$ & Y13 & Geißler et al. (2012) \\
\hline SS Comparison Sample & & & & \\
\hline \multicolumn{7}{l}{ ID } & Mass $\left(M_{\text {Jup }}\right)$ & Sep (au) & Survey $^{2}$ & Reference $^{3}$ \\
\hline AB Pic B & 13.5 & 275 & BN13 & Chauvin et al. (2005) \\
& And b & $14_{-2}^{+25}$ & $55 \pm 2$ & B14 & Carson et al. (2013) \\
& Tel B & $20-50$ & 185 & BN13 & Lowrance et al. (2000) \\
CD-35 2722 b & $31 \pm 8$ & $67 \pm 4$ & BN13 & Wahhaj et al. (2011) \\
HD 23514 b & $60 \pm 10$ & 360 & Y13 & Rodriguez et al. (2012) \\
PZ Tel b & $62 \pm 9$ & 20 & BN13 & Biller et al. (2010) \\
\hline
\end{tabular}

Notes. ${ }^{(1)}$ Reference number from Table B.1. ${ }^{(2)}$ Original Survey, from Table $1 .{ }^{(3)}$ Reference for the companion parameters.

the limits of the field of view of the imaging surveys considered here.

Table 3 summarises the characteristics of the detected companions in both the CBIN and the SS samples.

We used the approach described in Sect. 4.1 and the detection probability $\left(f p_{j}\right)$ evaluated as in Sect. 4.3 to constraint the frequency $f$ of sub-stellar companion in wide circumbinary orbits around the targets.

For a given value $f$ of the fraction of stars having at least one companion in the chosen range of mass and semi-major axis, we inverted Eq. (5) to estimate its probability $p\left(f \mid\left\{d_{j}\right\}\right)$.

Table 4 summarises the results we obtained for different choices of mass and semi-major axis ranges, for both the CBIN and the SS sample. Figure 3 shows the results obtained considering semi-major axis up to $1000 \mathrm{au}$.

For each case, Eqs. (7) and (6) were also used to calculate the values of $f_{\min }$ and $f_{\max }$ respectively, for a CL value of $68 \%$ and $95 \%$.

\section{Discussion}

\subsection{The frequency of planets and brown dwarfs in circumbinary orbits}

We have presented the results of the statistical analysis of a sample of 117 tight binaries observed in the contest of some of the deepest DI planet search surveys. Five of the targets included in our sample have reported detection of sub-stellar companions, two of which (HIP $59960 \mathrm{~b}$ and 2MASS J01033563-5515561 b) are in the planetary mass regime.

We find that our data are compatible with $6 \%$ (with an upper limit of $\sim 13 \%$ at $95 \%$ confidence level) of tight binaries hosting sub-stellar companions $\left(2 M_{\text {Jup }}<M_{\mathrm{c}}<70 M_{\text {Jup }}\right)$ within $1000 \mathrm{au}$. If we limit our analysis to planetary mass companions ( $2 M_{\text {Jup }}<M_{\mathrm{c}}<15 M_{\text {Jup }}$ ), the best frequency value is $2.70 \%$ (with a 95\% CL upper limit of $~ 9 \%$ ), for a semi-major axis cutoff of $1000 \mathrm{au}$, and $1.35 \%$ (with $f_{\max } \sim 7 \%$ at 95\% CL) for separations up to $100 \mathrm{au}$. 
Table 4. Statistical analysis results.

\begin{tabular}{cr|cccc|cccc}
\hline \hline \multicolumn{9}{c}{ CBIN sample } & \multicolumn{4}{c}{ SS comparison sample } \\
\hline SMA & \multicolumn{1}{c|}{ Mass } & $N_{\text {det }}^{1}$ & $f_{\text {best }}^{2}$ & \multicolumn{2}{c|}{$\left[f_{\min }, f_{\max }\right]^{3}$} & $N_{\text {det }}^{1}$ & $f_{\text {best }}^{2}$ & \multicolumn{2}{c}{$\left[f_{\min }, f_{\max }^{3}\right]$} \\
$(\mathrm{au})$ & $\left(M_{\text {Jup }}\right)$ & & $(\%)$ & $\mathrm{CL}=68 \%$ & $\mathrm{CL}=95 \%$ & & $(\%)$ & $\mathrm{CL}=68 \%$ & $\mathrm{CL}=95 \%$ \\
\hline $10-100$ & $2-15$ & 1 & 1.35 & {$[0.95,4.30]$} & {$[0.35,7.20]$} & 1 & 0.90 & {$[0.65,2.85]$} & {$[0.25,4.80]$} \\
& $15-70$ & 0 & - & {$[0.00,1.95]$} & {$[0.00,3.85]$} & 2 & 1.20 & {$[0.85,2.70]$} & {$[0.40,4.20]$} \\
& $2-70$ & 1 & 1.15 & {$[0.80,3.60]$} & {$[0.30,6.05]$} & 3 & 1.90 & {$[1.35,3.70]$} & {$[0.70,5.45]$} \\
\hline $10-500$ & $2-15$ & 1 & 1.30 & {$[0.95,4.10]$} & {$[0.35,6.85]$} & 2 & 1.60 & {$[1.10,3.60]$} & {$[0.50,5.60]$} \\
& $15-70$ & 3 & 3.30 & {$[2.30,6.30]$} & {$[1.20,9.25]$} & 4 & 2.50 & {$[1.80,4.40]$} & {$[1.05,6.25]$} \\
& $2-70$ & 4 & 4.50 & {$[3.20,7.80]$} & {$[1.85,11.00]$} & 6 & 3.95 & {$[2.95,6.15]$} & {$[1.90,8.35]$} \\
\hline $10-1000$ & $2-15$ & 2 & 2.70 & {$[1.85,6.00]$} & {$[0.85,9.25]$} & 2 & 1.85 & {$[1.30,4.20]$} & {$[0.60,6.55]$} \\
& $15-70$ & 3 & 3.55 & {$[2.50,6.75]$} & {$[1.30,9.90]$} & 4 & 3.05 & {$[2.20,5.30]$} & {$[1.25,7.55]$} \\
& $2-70$ & 5 & 6.00 & {$[4.35,9.75]$} & {$[2.70,13.35]$} & 6 & 4.70 & {$[3.50,7.35]$} & {$[2.25,9.95]$} \\
\hline
\end{tabular}

Notes. ${ }^{(1)}$ Number of detections in the considered mass and semi-major axis (SMA) range. ${ }^{(2)}$ Best value of the planet frequency compatible with the observations. ${ }^{(3)}$ Minimum and maximum values of the frequency compatible with the results, for a given confidence level (CL).

From a similar analysis of the SS control sample described in Sect. 2.2 we were able to infer a frequency of companions within 1000 au between $0.6 \%$ and $6.55 \%$ for the planetary mass objects and between $2.25 \%$ and $9.95 \%$ for companions up to $70 M_{\text {Jup }}$, within the same semi-major axis range.

Although our results seem to point towards the existence of small differences between the frequency of sub-stellar companions around close binaries and isolated stars, the significance of such result is only marginal (at most $2 \sigma$ for the 1000 au case, as shown also in Fig. 3) and needs confirmation through deeper observations and using larger samples.

Furthermore, possible selection effects may play a role. In particular, the discovery of sub-stellar objects around a target may have triggered dedicated follow-up observations resulting in improved sensitivity to very close stellar companions. This is likely the case of HIP 59960, while the other stellar companions of stars in Table 4 were known in advance or presented in the discovery papers of the sub-stellar companions. Our results therefore seem to suggest that no strong difference exists, in terms of frequency of sub-stellar companions in wide orbit, between close binaries and single stars.

\subsection{Comparison with Kepler results}

Welsh et al. (2012) estimated a frequency or circumbinary planets of about $3 \%$ (with lower limit of $1 \%$ ) when considering the short-period circumbinary planets detectable by Kepler. The separation range we are sensitive with direct imaging is different from that explored by Kepler and then the two techniques are highly complementary. Very recently, a circumbinary planet at 2.7 au was discovered with Kepler (Kostov et al. 2016), indicating that circumbinary planets likely are present over an extended separation range.

Some additional interesting trends are also emerging from the Kepler sample. Welsh et al. (2014) noticed the complete absence of transiting circumbinary planets around binaries with $p<5 \mathrm{~d}$. This seems unlikely to be due to selection effects. Indeed, according to Slawson et al. (2011) a relatively high number of these systems were in fact observed by Kepler. Moreover, such planets, as long as they are near the inner stability limit, would have an higher transit probability, and therefore be easy to detect. The lack of planets around very close binaries could be due to the formation history of the tight pair, which may be linked to the presence of an outer stellar companions which shrunk the central binary orbit via Kozai mechanism and tidal circularization (Martin et al. 2015). Sanz-Forcada et al. (2014) suggest strong photoevaporation, expected for this kind of tight binaries which keep fast rotation and high levels of magnetic activity for their whole lifetime, as a possible explanation for this lack of planets.

Our sample includes a large variety of binary configuration, with a fraction of binaries with very short periods (17\%), a number of binaries with orbital periods comparable to those of the hosts of Kepler circumbinary planets (7-41 days) and a significant number of wider binaries. Therefore, the possible lack of planets around very close pairs due to dynamical interaction has not a dominant role in our statistical analysis. Unfortunately, the binary properties of systems with detected sub-stellar companions are poorly constrained (orbits not available) for HIP 19176, HII 1348, 2MASS J01033563-5515561, and HIP 59960, while a reliable orbital solution was derived for TWA 5. However, a very close system is possible only for HII 1348.

Another property emerging from Kepler results is that often the circumbinary planets are found close to the dynamical stability limits. This is likely due to stopping of inward migration close to the inner disk limits caused by the presence of central binary (see e.g. Pierens \& Nelson 2013). The circumbinary sub-stellar objects identified with direct imaging are typically very far from the dynamical stability limits with only 2MASS J01033563$5515561 \mathrm{~b}$ being at a separation which is less than two times the adopted dynamical stability limit. This holds both for the objects included in the sample as well as for other circumbinary planets or brown dwarfs which are not included in our statistical analysis due to the lack of suitable publication of the parent sample such as Ross 458 (Burgasser et al. 2010) and FW Tau and ROX42B (Kraus et al. 2014) or because the binary is wider than our adopted limit, as SR12 (Kuzuhara et al. 2011). This could be explained by a different formation mechanism but ejection to outer orbits due to gravitational encounters is also a viable possibility. The system around HIP 59960 is of special interest in this context, thanks to the presence of both a circumbinary companion of planetary mass at wide separation and of a circumbinary disk which have been recently spatially resolved with SPHERE and GPI (Lagrange et al. 2016; Kalas et al. 2015). The on-going extension of the SPOTS program with SPHERE at VLT, probing closer separations, will be crucial for a better understanding of the separation distribution of circumbinary sub-stellar objects. 

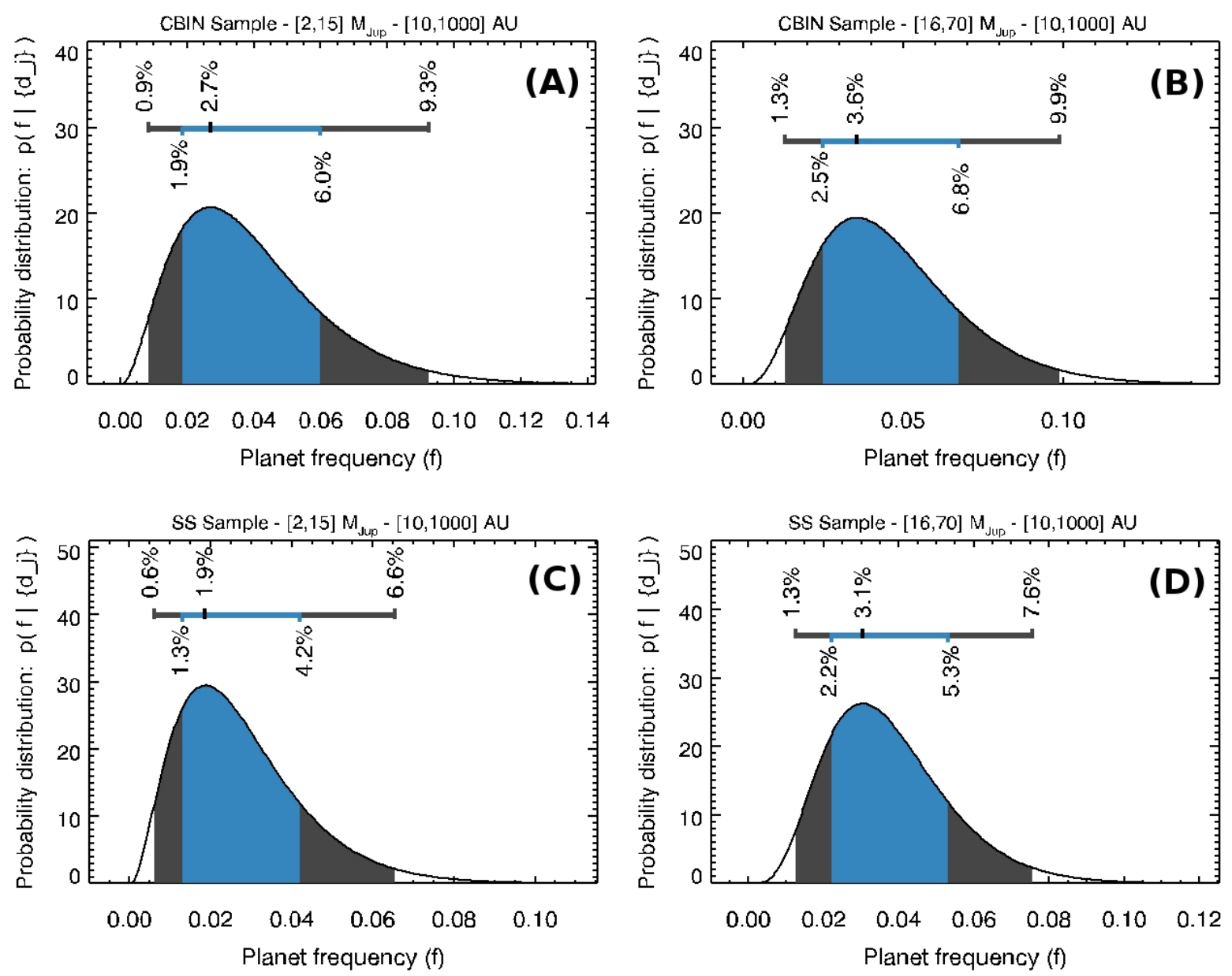

Fig. 3. Probability distribution (see Table 4 for details) of the frequency of planetary mass (up to $15 M_{\text {Jup }}$, left panels) and BD (16-70 $M_{\text {Jup }}$, right panels). The results for the CBIN sample and the and the SS control sample are shown in the upper and lower panels, respectively. The shaded areas show the frequency limits for the $95 \%$ (gray) and 68\% (blue) confidence levels.

\subsection{Implications for the origin of planet candidates around post-common envelope binaries}

In the past years, several claims of massive planetary companions orbiting post-common envelope binaries, based on the transit timing technique, appeared in the literature (Zorotovic \& Schreiber 2013, and references therein). Their existence is currently controversial, as in several cases the continuation of the observations did not follow the ephemeris from the discovery papers, calling for a full revision of the orbital elements and/or the inclusion of additional objects (see, e.g. Parsons et al. 2010; Beuermann et al. 2012). In other cases, the proposed multi-planet systems are not dynamically stable (see, e.g., Horner et al. 2013). Only the system orbiting NN Ser appears to be confirmed (Parsons et al. 2014), as timing variations are consistent with circumbinary planets for both the primary and secondary eclipses. The recent imaging non-detection of the brown dwarf candidate identified with timing technique around V471 Tau (Hardy et al. 2015) further calls into question the Keplerian origin of the observed eclipse timing variation (see however Vaccaro et al. 2015, for a different interpretation of the imaging non-detection).

If the observed timing variations are due to circumbinary planets, there are two paths for their formation. The first one is that they formed together with the central binary and survived the common envelope evolution of central pair (first generation scenario). In most cases, the observed wide separation could be compatible with this possibility. The second scenario is that circumbinary planets formed after the common envelope evolution, in the circumbinary disk that is expected to form from the material lost in the process. The large content of heavy elements expected in such disks (Waters et al. 1998) could contribute in a large efficiency of planet formation process in these environments. This scenario is favoured in the discussion by Zorotovic \& Schreiber (2013) and, for the specific case of the NN Ser system, by Mustill et al. (2013), while Bear \& Soker (2014) identified some difficulties with the second-generation model.

The first attempt to estimate the frequency of circumbinary planets around post-common envelope binaries was performed by Zorotovic \& Schreiber (2013). They found a very high frequency (90\% from 10 systems with adequate time baseline and measurement accuracy) of the occurrence of eclipse timing variations suggesting the presence of circumbinary planets. In most cases, these candidate companions are moderately massive $\left(5-10 \mathrm{M}_{\mathrm{J}}\right)$ and at moderately wide separation (5-10 au), i.e. within the mass and separation range we are probing with direct imaging (although the binary evolution could have caused some outward migration due to system mass loss). The similar (and relatively low) frequency of sub-stellar objects around 
close binaries and single stars found in our work points against the first-generation scenario being responsible for the majority of planet candidates around post-common-envelope binaries. This leaves as the most probable interpretations to the eclipsing timing variations either second generation planet formation or some non-Keplerian physical mechanisms mimicking the timing signature of planetary companions. It should be noticed that second generation planets are expected to be much younger than the age of the system and thus significantly brighter than 1st generation ones. This would strongly favour their direct detection. In the case of the NN Ser system, the cooling age of the white dwarf in the system is estimated to be just 1 Myr (Beuermann et al. 2010). We note that in the three cases of post-common envelope systems in our sample (Algol, Regulus, $\theta$ Hya), the detection limits were derived for the original system age, and thus are valid for first generation planets. Lower mass limits could be derived for planets formed at the time of the common-envelope evolution.

\section{Summary and conclusions}

We have presented a statistical analysis of the combined body of existing high-contrast imaging constraints on circumbinary planets, to complement our ongoing SPOTS direct imaging survey dedicated to such planets. The sample of stars considered includes 117 objects and comes from a search for tight binaries within the target lists of 23 published direct imaging surveys, including some of the deepest ones performed to data. This resulted in a large variety of binary configurations, including systems with very short periods, a number of binaries with orbital periods comparable to those of the hosts of Kepler circumbinary planets and a significant number of wider binaries.

The main conclusion of this work is the suggestion that no strong difference exists, in terms of frequency of sub-stellar companions in wide orbit, between close binaries and single stars.

With five of the pairs included in our circumbinary sample hosting sub-stellar companions, only two of which have planetary mass, we were able to constraint the frequency of circumbinary companions in wide orbits $(<1000 \mathrm{au})$ to a value between $\sim 0.9 \%$ and $\sim 9 \%$ for the planetary mass companions, and between $1.3 \%$ and $\sim 10 \%$ for low-mass brown dwarfs, with a confidence level of $95 \%$.

A similar analysis for the comparison sample of 205 single stars lead to a value of the frequency of planetary (low-mass BD) companions between $0.6 \%$ and $6.55 \%$ (1.25\% and $7.55 \%$ ), with the same confidence level.

Although there seem to be some small differences between the results for the two samples, the retrieved values of the frequency are compatible within the errors, and given the small number of target considered, it is premature to speculate about possible differences in the overall frequency, as well as in the formation mechanisms.

The similar (and relatively low) frequency of sub-stellar objects around close binaries and single stars also points against the first-generation scenario being responsible for the high abundance of planet candidates around post-common-envelope binaries.

This leaves as the most probable interpretations to the eclipsing timing variations observed in the majority of post-common envelope binaries either second generation planet formation or some non-Keplerian physical mechanisms mimicking the timing signature of planetary companions.

Our result nicely complement those coming from the Kepler spacecraft, as the separation range explored with direct imaging is quite different. Kepler's circumbinary planets are often close to the dynamical stability limit, whereas most the companions identified with direct imaging are instead much further out.

The on-going extension of the SPOTS program with SPHERE at VLT, probing closer separations, will be crucial for a better understanding of the separation distribution of circumbinary sub-stellar objects.

Acknowledgements. This research has made use of the SIMBAD database and of the VizieR catalogue access tool operated at CDS, Strasbourg, France, and of the Washington Double Star Catalogue maintained at the US Naval Observatory. We made use of data products retrieved from ESO Science Archive Facility (program 084.A-9004) and SOPHIE archive. The authors warmly thank AnneMarie Lagrange for sharing results in advance of publication. The authors would like to thank Beth Biller, Brendan Bowler, Alexis Brandeker, Sebastian Daemgen, Philippe Delorme, Markus Kasper, David Lafreniere, Anne-Lise Maire and Inseok Song for providing the detection limits and the target information from their surveys. We thank the anonymous referee for extensive feedback that significantly improved the clarity of the paper. S.D. acknowledges support from the "Progetti Premiali" funding scheme of the Italian Ministry of Education, University, and Research.

\section{References}

Africano, J. L., Cobb, C. L., Dunham, D. W., et al. 1975, ApJ, 80, 689 Alecian, E., Catala, C., van't Veer-Menneret, C., Goupil, M.-J., \& Balona, L. 2005, A\&A, 442, 993

Allard, F., Hauschildt, P. H., Alexander, D. R., Tamanai, A., \& Schweitzer, A. 2001, ApJ, 556, 357

Alonso, R., Deeg, H. J., Hoyer, S., et al. 2015, A\&A, 584, L8

Andrews, S. M., \& Williams, J. P. 2005, ApJ, 619, L175

Arriagada, P. 2011, ApJ, 734, 70

Bailey, III, J. I., White, R. J., Blake, C. H., et al. 2012, ApJ, 749, 16

Bailey, V., Meshkat, T., Reiter, M., et al. 2014, ApJ, 780, L4

Baraffe, I., Chabrier, G., Barman, T. S., Allard, F., \& Hauschildt, P. H. 2003, A\&A, 402, 701

Barnes, S. A. 2007, ApJ, 669, 1167

Barrado y Navascués, D., Stauffer, J. R., \& Jayawardhana, R. 2004, ApJ, 614, 386

Bear, E., \& Soker, N. 2014, MNRAS, 444, 1698

Bell, C. P. M., Mamajek, E. E., \& Naylor, T. 2015, MNRAS, 454, 593

Beuermann, K., Hessman, F. V., Dreizler, S., et al. 2010, A\&A, 521, L60

Beuermann, K., Dreizler, S., Hessman, F. V., \& Deller, J. 2012, A\&A, 543, A138

Beuzit, J.-L., Boccaletti, A., Feldt, M., et al. 2010, in Pathways Towards

Habitable Planets, eds. V. Coudé du Foresto, D. M. Gelino, \& I. Ribas, ASP

Conf. Ser., 430, 231

Biller, B. A., Close, L. M., Masciadri, E., et al. 2007, ApJS, 173, 143

Biller, B. A., Liu, M. C., Wahhaj, Z., et al. 2010, ApJ, 720, L82

Biller, B. A., Liu, M. C., Wahhaj, Z., et al. 2013, ApJ, 777, 160

Bobylev, V. V., Goncharov, G. A., \& Bajkova, A. T. 2007, VizieR Online Data Catalog: J/PAZh/32/906

Bonavita, M., \& Desidera, S. 2007, A\&A, 468, 721

Bonavita, M., Chauvin, G., Desidera, S., et al. 2012, A\&A, 537, A67

Bonavita, M., de Mooij, E. J. W., \& Jayawardhana, R. 2013, PASP, 125, 849

Bonavita, M., Daemgen, S., Desidera, S., et al. 2014, ApJ, 791, L40

Bonnefoy, M., Chauvin, G., Dumas, C., et al. 2009, A\&A, 506, 799

Booth, M., Kennedy, G., Sibthorpe, B., et al. 2013, MNRAS, 428, 1263

Bouchy, F., Hébrard, G., Udry, S., et al. 2009, A\&A, 505, 853

Bowler, B. P., Liu, M. C., Shkolnik, E. L., \& Tamura, M. 2015, ApJS, 216, 7

Brandeker, A., Jayawardhana, R., Khavari, P., Haisch, Jr., K. E., \& Mardones, D. 2006, ApJ, 652, 1572

Brandt, T. D., Kuzuhara, M., McElwain, M. W., et al. 2014a, ApJ, 786, 1

Brandt, T. D., McElwain, M. W., Turner, E. L., et al. 2014b, ApJ, 794, 159

Bubar, E. J., King, J. R., Soderblom, D. R., Deliyannis, C. P., \& Boesgaard, A. M. 2007, ApJ, 134, 2328

Burgasser, A. J., Simcoe, R. A., Bochanski, J. J., et al. 2010, ApJ, 725, 1405

Burleigh, M. R., \& Barstow, M. A. 1999, A\&A, 341, 795

Buscombe, W., \& Morris, P. M. 1961, MNRAS, 123, 233

Carson, J., Thalmann, C., Janson, M., et al. 2013, ApJ, 763, L32

Catalán, S., Ribas, I., Isern, J., \& García-Berro, E. 2008, A\&A, 477, 901

Chauvin, G., Lagrange, A.-M., Zuckerman, B., et al. 2005, A\&A, 438, L29

Chauvin, G., Lagrange, A.-M., Bonavita, M., et al. 2010, A\&A, 509, A52

Chauvin, G., Vigan, A., Bonnefoy, M., et al. 2015, A\&A, 573, A127

Chen, C. H., Mamajek, E. E., Bitner, M. A., et al. 2011, ApJ, 738, 122

Close, L. M., Lenzen, R., Guirado, J. C., et al. 2005, Nature, 433, 286

Cutispoto, G., Pastori, L., Pasquini, L., et al. 2002, A\&A, 384, 491 
Daemgen, S., Bonavita, M., Jayawardhana, R., Lafrenière, D., \& Janson, M 2015, ApJ, 799, 155

Dahm, S. E., Slesnick, C. L., \& White, R. J. 2012, ApJ, 745, 56

da Silva, L., Torres, C. A. O., de La Reza, R., et al. 2009, A\&A, 508, 833

Delorme, P., Lagrange, A. M., Chauvin, G., et al. 2012, A\&A, 539, A72

Delorme, P., Gagné, J., Girard, J. H., et al. 2013, A\&A, 553, L5

De Rosa, R. J., Patience, J., Wilson, P. A., et al. 2014, MNRAS, 437, 1216

Desidera, S., Covino, E., Messina, S., et al. 2015, A\&A, 573, A126

Dommanget, J., \& Nys, O. 2002, VizieR Online Data Catalog: I/274

Duchêne, G. 2010, ApJ, 709, L114

Eisenbeiss, T., Ammler-von Eiff, M., Roell, T., et al. 2013, A\&A, 556, A53

Elliott, P., Bayo, A., Melo, C. H. F., et al. 2014, A\&A, 568, A26

Evans, T. M., Ireland, M. J., Kraus, A. L., et al. 2012, ApJ, 744, 120

Favata, F., Barbera, M., Micela, G., \& Sciortino, S. 1995, A\&A, 295, 147

Favata, F., Micela, G., \& Sciortino, S. 1996, A\&A, 311, 951

Feigelson, E. D., Lawson, W. A., Stark, M., Townsley, L., \& Garmire, G. P. 2006, ApJ, 131, 1730

Fekel, F. C., Barlow, D. J., Scarfe, C. D., Jancart, S., \& Pourbaix, D. 2005, AJ, 129,1001

Fekel, F. C., Williamson, M., Buggs, C., Onuoha, G., \& Smith, B. 2006, ApJ 132,1490

Fortney, J. J., Marley, M. S., Saumon, D., \& Lodders, K. 2008, ApJ, 683, 1104

Fuhrmann, K. 2008, MNRAS, 384, 173

Galland, F., Lagrange, A.-M., Udry, S., et al. 2005, A\&A, 443, 337

Gálvez, M. C., Montes, D., Fernández-Figueroa, M. J., \& López-Santiago, J. 2006, Ap\&SS, 304, 59

Geißler, K., Metchev, S. A., Pham, A., et al. 2012, ApJ, 746, 44

Ghez, A. M., Neugebauer, G., \& Matthews, K. 1993, AJ, 106, 2005

Gies, D. R., Dieterich, S., Richardson, N. D., et al. 2008, ApJ, 682, L117

Goldin, A., \& Makarov, V. V. 2007, ApJS, 173, 137

Gray, R. O., Corbally, C. J., Garrison, R. F., et al. 2006, ApJ, 132, 161

Griffin, R. F. 2010, The Observatory, 130, 125

Griffin, R. F., \& Filiz Ak, N. 2010, Ap\&SS, 330, 47

Guenther, E. W., \& Esposito, E. 2007, ArXiv e-prints

[arXiv:astro-ph/0701293]

Guenther, E. W., Esposito, M., Mundt, R., et al. 2007, A\&A, 467, 1147

Guirado, J. C., Martí-Vidal, I., Marcaide, J. M., et al. 2006, A\&A, 446, 733

Haghighipour, N. 2010, Planets in Binary Star Systems, Astrophys. Space Sci. Libr., 366

Hardy, A., Schreiber, M. R., Parsons, S. G., et al. 2015, ApJ, 800, L24

Heinze, A. N., Hinz, P. M., Sivanandam, S., et al. 2010, ApJ, 714, 1551

Hełminiak, K. G., Brahm, R., Ratajczak, M., et al. 2014, A\&A, 567, A64

Henry, T. J., Franz, O. G., Wasserman, L. H., et al. 1999, ApJ, 512, 864

Hinkley, S., Kraus, A. L., Ireland, M. J., et al. 2015, ApJ, 806, L9

Holberg, J. B., Oswalt, T. D., Sion, E. M., Barstow, M. A., \& Burleigh, M. R. 2013, MNRAS, 435, 2077

Holman, M. J., \& Wiegert, P. A. 1999, AJ, 117, 621

Holmberg, J., Nordström, B., \& Andersen, J. 2009, A\&A, 501, 941

Hormuth, F., Brandner, W., Hippler, S., Janson, M., \& Henning, T. 2007, A\&A, 463, 707

Horner, J., Wittenmyer, R. A., Hinse, T. C., et al. 2013, MNRAS, 435, 2033

Janson, M., Brandner, W., Lenzen, R., et al. 2007, A\&A, 462, 615

Janson, M., Bonavita, M., Klahr, H., et al. 2011, ApJ, 736, 89

Janson, M., Hormuth, F., Bergfors, C. et al. 2012, ApJ, 754, 44

Janson, M., Brandt, T. D., Moro-Martín, A., et al. 2013a, ApJ, 773, 73

Janson, M., Lafrenière, D., Jayawardhana, R., et al. 2013b, ApJ, 773, 170

Janson, M., Thalmann, C., Boccaletti, A., et al. 2016, ApJ, 816, L1

Jayawardhana, R., Coffey, J., Scholz, A., Brandeker, A., \& van Kerkwijk, M. H. 2006, ApJ, 648, 1206

Joergens, V., Janson, M., \& Müller, A. 2012, A\&A, 537, A13

Kalas, P. G., Rajan, A., Wang, J. J., et al. 2015, ApJ, 814, 32

Kasper, M., Apai, D., Janson, M., \& Brandner, W. 2007, A\&A, 472, 321

King, J. R., Villarreal, A. R., Soderblom, D. R., Gulliver, A. F., \& Adelman, S. J. 2003, AJ, 125, 1980

Kiraga, M. 2012, Acta Astron., 62, 67

Kirkpatrick, J. D., Dahn, C. C., Monet, D. G., et al. 2001, AJ, 121, 3235

Koen, C., \& Eyer, L. 2002, MNRAS, 331, 45

Köhler, R. 2011, A\&A, 530, A126

Köhler, R., Kunkel, M., Leinert, C., \& Zinnecker, H. 2000, A\&A, 356, 541

Köhler, R., Ratzka, T., Petr-Gotzens, M. G., \& Correia, S. 2013, A\&A, 558, A80

Konopacky, Q. M., Ghez, A. M., Duchêne, G., McCabe, C., \& Macintosh, B. A 2007, ApJ, 133, 2008

Kostov, V. B., Orosz, J. A., Welsh, W. F., et al. 2016, ApJ, 827, 86

Kraus, A. L., Ireland, M. J., Martinache, F., \& Lloyd, J. P. 2008, ApJ, 679, 762

Kraus, A. L., Ireland, M. J., Cieza, L. A., et al. 2014, ApJ, 781, 20

Kuzuhara, M., Tamura, M., Ishii, M., et al. 2011, AJ, 141, 119

Kuzuhara, M., Tamura, M., Kudo, T., et al. 2013, ApJ, 774, 11

Lafrenière, D., Doyon, R., Marois, C., et al. 2007, ApJ, 670, 1367
Lafrenière, D., Jayawardhana, R., van Kerkwijk, M. H., Brandeker, A., \& Janson, M. 2014, ApJ, 785, 47

Lagrange, A.-M., Bonnefoy, M., Chauvin, G., et al. 2010, Science, 329, 57

Lagrange, A.-M., Langlois, M., Gratton, R., et al. 2016, A\&A, 586, L8

Lannier, J., Delorme, P., Lagrange, A. M., et al. 2016, A\&A, in press, DOI: 10.1051/0004-6361/201628237

Latham, D. W., Stefanik, R. P., Torres, G., et al. 2002, ApJ, 124, 1144

Levato, H., Malaroda, S., Morrell, N., \& Solivella, G. 1987, ApJS, 64, 487

Liu, T., Janes, K. A., \& Bania, T. M. 1991, ApJ, 377, 141

López-Santiago, J., Montes, D., Gálvez-Ortiz, M. C., et al. 2010, A\&A, 514, A97

Lowrance, P. J., McCarthy, C., Becklin, E. E., et al. 1999, ApJ, 512, L69

Lowrance, P. J., Schneider, G., Kirkpatrick, J. D., et al. 2000, ApJ, 541, 390

Lowrance, P. J., Becklin, E. E., Schneider, G., et al. 2005, AJ, 130, 1845

Luhman, K. L., Patten, B. M., Marengo, M., et al. 2007, ApJ, 654, 570

Macintosh, B., Max, C., Zuckerman, B., et al. 2001, in Young Stars Near Earth:

Progress and Prospects, eds. R. Jayawardhana, \& T. Greene, ASP Conf. Ser., 244, 309

Maire, A.-L., Boccaletti, A., Rameau, J., et al. 2014, A\&A, 566, A126

Makarov, V. V., \& Kaplan, G. H. 2005, ApJ, 129, 2420

Makarov, V. V., Zacharias, N., Hennessy, G. S., Harris, H. C., \& Monet, A. K. B. 2007, ApJ, 668, L155

Malo, L., Doyon, R., Lafrenière, D., et al. 2013, ApJ, 762, 88

Malo, L., Artigau, É., Doyon, R., et al. 2014, ApJ, 788, 81

Marley, M. S., Fortney, J. J., Hubickyj, O., Bodenheimer, P., \& Lissauer, J. J. 2007, ApJ, 655, 541

Marois, C., Zuckerman, B., Konopacky, Q. M., Macintosh, B., \& Barman, T. 2010, Nature, 468, 1080

Martin, D. V., Mazeh, T., \& Fabrycky, D. C. 2015, MNRAS, 453, 3554

Mason, B. D. 1996, ApJ, 112, 2260

Mathieu, R. D., Walter, F. M., \& Myers, P. C. 1989, AJ, 98, 987

Mathieu, R. D., Stassun, K., Basri, G., et al. 1997, AJ, 113, 1841

Mishenina, T. V., Soubiran, C., Kovtyukh, V. V., Katsova, M. M., \& Livshits, M. A. 2012, A\&A, 547, A106

Montes, D., López-Santiago, J., Fernández-Figueroa, M. J., \& Gálvez, M. C. 2001a, A\&A, 379, 976

Montes, D., López-Santiago, J., Gálvez, M. C., et al. 2001b, MNRAS, 328, 45

Moór, A., Szabó, G. M., Kiss, L. L., et al. 2013, MNRAS, 435, 1376

Morales, F. Y., Rieke, G. H., Werner, M. W., et al. 2011, ApJ, 730, L29

Mustill, A. J., Marshall, J. P., Villaver, E., et al. 2013, MNRAS, 436, 2515

Neuhäuser, R., Guenther, E. W., Alves, J., et al. 2003, Astron. Nachr., 324, 535

Neuhäuser, R., Schmidt, T. O. B., Hambaryan, V. V., \& Vogt, N. 2010, A\&A, 516, A112

Nguyen, D. C., Brandeker, A., van Kerkwijk, M. H., \& Jayawardhana, R. 2012, ApJ, 745, 119

Nidever, D. L., Marcy, G. W., Butler, R. P., Fischer, D. A., \& Vogt, S. S. 2002, ApJS, 141, 503

Nielsen, E. L., Liu, M. C., Wahhaj, Z., et al. 2013, ApJ, 776, 4

Nordström, B., Mayor, M., Andersen, J., et al. 2004, A\&A, 418, 989

Pallavicini, R., Pasquini, L., \& Randich, S. 1992a, A\&A, 261, 245

Pallavicini, R., Randich, S., \& Giampapa, M. S. 1992b, A\&A, 253, 185

Parsons, S. G., Marsh, T. R., Copperwheat, C. M., et al. 2010, MNRAS, 407, 2362

Parsons, S. G., Marsh, T. R., Bours, M. C. P., et al. 2014, MNRAS, 438, L91

Pecaut, M. J., Mamajek, E. E., \& Bubar, E. J. 2012, ApJ, 746, 154

Perryman, M. A. C., \& ESA, 1997, in The HIPPARCos and TYCHO catalogues. Astrometric and photometric star catalogues derived from the ESA HIPPARcos Space Astrometry Mission, ESA SP, 1200

Pierens, A., \& Nelson, R. P. 2013, A\&A, 556, A134

Pizzolato, N., Maggio, A., Micela, G., Sciortino, S., \& Ventura, P. 2003, A\&A, 397,147

Plavchan, P., Werner, M. W., Chen, C. H., et al. 2009, ApJ, 698, 1068

Pourbaix, D., Tokovinin, A. A., Batten, A. H., et al. 2004, A\&A, 424, 727

Queloz, D., Allain, S., Mermilliod, J.-C., Bouvier, J., \& Mayor, M. 1998, A\&A, 335,183

Raghavan, D., McAlister, H. A., Henry, T. J., et al. 2010, ApJS, 190, 1

Rameau, J., Chauvin, G., Lagrange, A.-M., et al. 2013a, ApJ, 772, L15

Rameau, J., Chauvin, G., Lagrange, A.-M., et al. 2013b, A\&A, 553, A60

Rappaport, S., Podsiadlowski, P., \& Horev, I. 2009, ApJ, 698, 666

Rhee, J. H., Song, I., Zuckerman, B., \& McElwain, M. 2007, ApJ, 660, 1556

Riaz, B., Gizis, J. E., \& Harvin, J. 2006, AJ, 132, 866

Ribas, I. 2003, A\&A, 400, 297

Richichi, A., Percheron, I., \& Khristoforova, M. 2005, A\&A, 431, 773

Richichi, A., Chen, W. P., Cusano, F., et al. 2012, A\&A, 541, A96

Riedel, A. R., Finch, C. T., Henry, T. J., et al. 2014, AJ, 147, 85

Rizzuto, A. C., Ireland, M. J., Robertson, J. G., et al. 2013, MNRAS, 436, 1694

Rodriguez, D. R., Marois, C., Zuckerman, B., Macintosh, B., \& Melis, C. 2012, ApJ, 748, 30 
M. Bonavita et al.: Constraints on the frequency of circumbinary planets in wide orbits

Rodriguez, D. R., Zuckerman, B., Kastner, J. H., et al. 2013, ApJ, 774, 101

Saar, S. H., Huovelin, J., Osten, R. A., \& Shcherbakov, A. G. 1997, A\&A, 326 741

Sanz-Forcada, J., Desidera, S., \& Micela, G. 2014, A\&A, 570, A50

Sarna, M. J. 1993, MNRAS, 262, 534

Sato, B., Fischer, D. A., Ida, S., et al. 2009, ApJ, 703, 671

Schlieder, J. E., Lépine, S., \& Simon, M. 2012, AJ, 144, 109

Schneider, J., Dedieu, C., Le Sidaner, P., Savalle, R., \& Zolotukhin, I. 2011 A\&A, 532, A79

Schröder, C., \& Schmitt, J. H. M. M. 2007, A\&A, 475, 677

Ségransan, D., Delfosse, X., Forveille, T., et al. 2000, A\&A, 364, 665

Shkolnik, E. L., Anglada-Escudé, G., Liu, M. C., et al. 2012, ApJ, 758, 56

Skemer, A., Apai, D., Bailey, V., et al. 2014, in IAU Symp. 299, eds. M. Booth, B. C. Matthews, \& J. R. Graham, 70

Slawson, R. W., Prša, A., Welsh, W. F., et al. 2011, AJ, 142, 160

Soderblom, D. R., King, J. R., \& Henry, T. J. 1998, AJ, 116, 396

Soderblom, D. R., Hillenbrand, L. A., Jeffries, R. D., Mamajek, E. E., \& Naylor, T. 2014, Protostars and Planets VI, 219

Song, I., Zuckerman, B., \& Bessell, M. S. 2003, ApJ, 599, 342

Song, I., Schneider, G., Zuckerman, B., et al. 2006, ApJ, 652, 724

Song, I., Zuckerman, B., \& Bessell, M. S. 2012, AJ, 144, 8

Stickland, D. J., Lloyd, C., Koch, R. H., \& Pachoulakis, I. 1996, The Observatory, 116, 387

Strassmeier, K., Washuettl, A., Granzer, T., Scheck, M., \& Weber, M. 2000, A\&AS, 142, 275

Teixeira, R., Ducourant, C., Chauvin, G., et al. 2009, A\&A, 503, 281

Thalmann, C., Carson, J., Janson, M., et al. 2009, ApJ, 707, L123

Thalmann, C., Desidera, S., Bonavita, M., et al. 2014, A\&A, 572, A91

Tokovinin, A. 2008, MNRAS, 389, 925

Tokovinin, A. 2013, ApJ, 145, 76

Tokovinin, A. 2014, AJ, 147, 86

Tokovinin, A., Thomas, S., Sterzik, M., \& Udry, S. 2006, A\&A, 450, 681

Tokovinin, A., Pribulla, T., \& Fischer, D. 2015, AJ, 149, 8

Tomkin, J., \& Popper, D. M. 1986, ApJ, 91, 1428
Torres, G., \& Ribas, I. 2002, ApJ, 567, 1140

Torres, G., Guenther, E. W., Marschall, L. A., et al. 2003, ApJ, 125, 825

Torres, C. A. O., Quast, G. R., da Silva, L., et al. 2006, A\&A, 460, 695

Torres, C. A. O., Quast, G. R., Melo, C. H. F., \& Sterzik, M. F. 2008, in Handbook of Star Forming Regions, Vol. II, 757

Tremko, J., Bakos, G. A., Žižňovský, J., \& Pribulla, T. 2010, Contributions of the Astronomical Observatory Skalnate Pleso, 40, 83

Vaccaro, T. R., Wilson, R. E., Van Hamme, W., \& Terrell, D. 2015, ApJ, 810, 157

Van Leeuwen, F. 2007, A\&A, 474, 653

Vennes, S., Christian, D. J., \& Thorstensen, J. R. 1998, ApJ, 502, 763

Vigan, A., Patience, J., Marois, C., et al. 2012, A\&A, 544, A9

Wahhaj, Z., Liu, M. C., Biller, B. A., et al. 2011, ApJ, 729, 139

Waters, L. B. F. M., Cami, J., de Jong, T., et al. 1998, Nature, 391, 868

Webb, R. A., Zuckerman, B., Platais, I., et al. 1999, ApJ, 512, L63

Weinberger, A. J., Anglada-Escudé, G., \& Boss, A. P. 2013, ApJ, 762, 118

Weis, E. W. 1991, AJ, 101, 1882

Welsh, W. F., Orosz, J. A., Carter, J. A., et al. 2012, Nature, 481, 475

Welsh, W. F., Orosz, J. A., Carter, J. A., \& Fabrycky, D. C. 2014, in IAU Symp. 293, ed. N. Haghighipour, 125

White, R. J., Gabor, J. M., \& Hillenbrand, L. A. 2007, ApJ, 133, 2524

Worley, C. E., \& Douglass, G. G. 1997, A\&AS, 125, 523

Wright, J. T., Marcy, G. W., Butler, R. P., \& Vogt, S. S. 2004, ApJS, 152, 261

Wright, J. T., Fakhouri, O., Marcy, G. W., et al. 2011, PASP, 123, 412

Yamamoto, K., Matsuo, T., Shibai, H., et al. 2013, PASJ, 65, 90

Zorotovic, M., \& Schreiber, M. R. 2013, A\&A, 549, A95

Zuckerman, B., \& Song, I. 2004, ARA\&A, 42, 685

Zuckerman, B., \& Webb, R. A. 2000, ApJ, 535, 959

Zuckerman, B., Song, I., \& Webb, R. A. 2001, ApJ, 559, 388

Zuckerman, B., Song, I., \& Bessell, M. S. 2004, ApJ, 613, L65

Zuckerman, B., Bessell, M. S., Song, I., \& Kim, S. 2006, ApJ, 649, L115

Zuckerman, B., Rhee, J. H., Song, I., \& Bessell, M. S. 2011, ApJ, 732, 61

Zuckerman, B., Vican, L., Song, I., \& Schneider, A. 2013, ApJ, 778, 5

Zurlo, A., Vigan, A., Hagelberg, J., et al. 2013, A\&A, 554, A21 


\section{Appendix A: Notes on individual objects}

1. TYC 5839-0596-1 See Desidera et al. (2015).

2. HIP 3210 Classified as SB2 from Nordström et al. (2004) with mass ratio of 0.35 . The orbital solution is not available. Moór et al. (2013) classified the star as a member of Columba association on the basis of the strength of lithium and kinematics, but without taking multiplicity into account. The system is also moderately X-ray bright. Considering the limited sensitivity of these age indicators for late F stars and the complications introduced by multiplicity, we adopt an age of 150 Myr. The possibility of tidal locking cannot be ruled out but the young disk kinematics and lithium would make an old age unlikely. The confirmation of Columba membership would require additional data on binary orbital solution.

3. HIP 3924 See Desidera et al. (2015).

4. $H I P 4448=H D 5578=B W$ Phe Classified as a new potential member of Tuc-Hor association in Zuckerman et al. (2001) (but not included in the list by Zuckerman \& Song 2004). Torres et al. (2008) and Malo et al. (2013) instead classified it a member of Argus association. The age indicators support a young age with upper limit of $150 \mathrm{Myr}$. We then adopt Argus membership and age, but stressing the uncertainty in the kinematic parameters due the unknown binary orbit. Indeed, the star is a close binary with similar components (projected separation 0.228 arcsec).

5. 2MASS J01033563-5515561 Close visual pair with a detected companion close to deuterium burning mass in circumbinary configuration (Delorme et al. 2013). The system is a probable member of Tuc-Hor association.

6. HIP $4967=G 132-50 A$ Young M dwarf, probable member of AB Dor MG, resolved into a tight binary by B15. There is an additional wide companion $\mathrm{G} 132-50 \mathrm{~B}$ at 25.6 arcsec, which it itself a 2 arcsec pair, making the system quadruple.

7. HIP $9141=H D 12039=$ DK Cet Member of Tucana association. A close stellar companion $(\rho=0.15$ arcsec) was imaged by Biller et al. (2007).

8. NLTT 6549 Young M dwarf, possible member of Hyades stream, resolved into a tight binary by B15. We adopt the parameters by $\mathrm{B} 15$.

9. HIP $11072=H D 14802=\kappa$ For Triple system, formed by a solar type star and a close pair of $\mathrm{M}$ dwarfs with tentative period of about 3 days. A full orbital solution of the outer orbit is available (Tokovinin 2013), including RV, astrometry and resolved imaging of the components. Isochrone fitting from Holmberg et al. (2009) gives $5.7 \pm 0.5$ Gyr, fully consistent with the low chromospheric emission reported from Wright et al. (2004) $\left(\log R_{H K}=-5.05\right)$. The $\mathrm{X}$ ray emission is instead larger, comparable to Hyades stars of similar colour, but this may be dominated by the emission from the close pair of $\mathrm{M}$ dwarfs due to their probable tidal locking. Barnes (2007) report a gyro-age of $730 \mathrm{Myr}$, from a rotation period of 9 days, that is wrong due to a typo in Pizzolato et al. (2003) (the referenced paper Saar et al. 1997, gives 19.3 days, derived from chromospheric emission). We then adopt the isochrone age.

10. $H I P 12413=H D 16754 A=s$ Eri Star with various signatures of multiplicity. As discussed in Zuckerman et al. (2011), the high-resolution X-ray imaging by Schröder \& Schmitt (2007) indicates that the early-type primary should have a spatially unresolved low mass companion. The presence of RV variations (Buscombe \& Morris 1961) and of the astrometric acceleration in HIPPARCos catalogue further support the binarity and suggest an orbital period of several years. We derive the stability limit for a semi-major axis of $5 \mathrm{au}$ and a mass of $0.6 M_{\odot}$. There is an additional M-type companion at 24 arcsec. The system is a probable member of Columba association (Zuckerman et al. 2011).

11. HIP $12545=B D+050378$ See Thalmann et al. (2014) Member of BPIC MG. Identified as SB1 in Song et al. (2003) (peak-to-valley variation of $20 \mathrm{~km} \mathrm{~s}^{-1}$, no orbital solution provided). However, Bailey et al. (2012) found no evidence for large RV variations from their monitoring over 600 days (14 epochs, scatter of $179 \mathrm{~m} / \mathrm{s}$ ).

12. HIP $12638=H D 16760$ Radial velocity monitoring revealed a sub-stellar companion of projected mass $m \sin i$ about 14 M (Sato et al. 2009; Bouchy et al. 2009). The direct detection by Evans et al. (2012) shows that the true mass is significantly larger than the minimum mass and that the inclination is very close to pole-on. Evans et al. (2012) derived a combined imaging and RV orbital solution, which we adopt in our study. Evans et al. (2012) also summarised the puzzling results from different age diagnostics. The adopted age is derived from the membership to AB Dor moving group. The star has a wide companion (HIP 12635) at 14 arcsec.

13. HIP $13081=H D 17382=B C$ Ari $=$ GJ 113 Triple system. The primary is a spectroscopic and astrometric binary (HIPPARCos acceleration). Latham et al. (2002) derived a preliminary spectroscopic orbital solution with period about $17 \mathrm{yr}$ in a rather eccentric orbit. The minimum mass of the companion is about $0.18 M_{\odot}$. There is also a wide companion $\left(\mathrm{GJ} 113 \mathrm{C}\right.$ ) at $20 \operatorname{arcsec}\left(\operatorname{mass} M_{B}=0.16 M_{\odot}\right.$ ). The star is a probable member of Hercules-Lyra according to Fuhrmann (2008). Activity indicators are consistent with a slightly older age (about $400 \mathrm{Myr}$ ) while lithium was not detected in the spectrum (Favata et al. 1996) suggesting an age of about 600 Myr or older. We then consider the membership unlikely, as also concluded by Eisenbeiss et al. (2013). The discrepancy between age indicators might also be explained if the unseen companion is actually white dwarf rather than a low mass main sequence star (see Zurlo et al. 2013, for the case of HD8049). But considering the lack of evidences supporting this latter hypothesis and the marginal amount of the discrepancy between age derived from lithium and activity indicators, we adopt an age $500 \mathrm{Myr}$.

14. HIP $14555=$ GJ 1054 A Short-period SB2 with similar components. See Maire et al. (2014).

15. HIP $14576=$ Algol $=H D 19356$ Triple system, with an inner pair evolved through mass transfer phase, and an additional component that is anyway close enough $(a=2.78 \mathrm{au})$ to allow the search for planets around the three stars. Stellar masses and orbital parameters from Sarna (1993).

16. HIP $16247=H D 21703=A K$ For Eclipsing binary recently studied by Hełminiak et al. (2014). The high levels of chromospheric and coronal activity are due to tidal locking and not to young age, as indicated by the lack of detection of lithium by Favata et al. (1995), that corresponds to a lower limit to stellar age of about $200 \mathrm{Myr}$. The thin disk kinematics is compatible with an age similar to that of the Sun.

17. 2MASS J03363144-2619578 = SCR J0336-2619 New close visual binary from Lannier et al. (2016); probable member of Tuc-Hor or Columba associations according to Rodriguez et al. (2013).

18. HIP $16853=$ HD 22705 See Thalmann et al. (2014).

19. HD 282954 SB2 in Pleiades open cluster according to Queloz et al. (1998). No orbit available. 
20. HII 1348 SB2 in Pleiades open cluster according to Queloz et al. (1998). No orbit available Individual masses 0.67 and $0.55 M_{\odot}$ from Geißler et al. (2012) Circumbinary brown dwarf detected by Geißler et al. (2012) and Yamamoto et al. (2013).

21. HD 23863 Close visual companion in Pleiades open cluster detected by Richichi et al. (2012) using the lunar occultation technique at a projected separation of $22.1 \mathrm{mas}=2.95 \mathrm{au}$. Estimated individual magnitudes are 7.60 and 1.66 in $K$ band, that, coupled with the distance and age of the Pleiades, lead to individual masses of 1.75 and $0.45 M_{\odot}$. The star is also a SB according to Liu et al. (1991). Richichi et al. (2012) were not able to conclude whether this is the same object responsible of the RV variations, due to the scarcity of the available info on the RV variations.

22. HIP $19176=H D 284149$ A brown dwarf companion was recently detected by Bonavita et al. (2014) at a projected separation of about $400 \mathrm{au}$. As discussed in this paper, the RV variability indicates the presence of an additional companion at small separation. We adopt the stellar parameters from Bonavita et al. (2014).

23. $R X J 0415.8+3100=V 952 \mathrm{Per}$ This star was classified as a short-period SB1 by Nguyen et al. (2012) on the basis of the large $\left(70 \mathrm{~km} \mathrm{~s}^{-1}\right) \mathrm{RV}$ variations over timescales of days. A lower limit to the companion mass is $0.21 M_{\odot}$ assuming a period of 2 days and a RV semi-amplitude of $35 \mathrm{~km} \mathrm{~s}^{-1}$. An additional component at 0.9 arcsec makes the system triple. Daemgen et al. (2015) classified the star as member of the Taurus Extended association. We estimated a distance of $200 \mathrm{pc}$ with a reddening $E(B-V)=0.15$, after correcting the system magnitude for the presence of the visual companion and assuming negligible flux contribution by the spectroscopic component. An age of about $100 \mathrm{Myr}$ is estimated from Lithium EW.

24. $R X$ J0435.9+2352 = V1324 Tau Close visual binary. D15 classified it in the Taurus extended group. We adopt an age of 20 Myr following D15.

25. HIP $21482=H D 283750=V 833$ Tau Triple system: V833 Tau is a spectroscopic binary with period 1.79 days. The mass of the companion has been estimate by Fuhrmann (2008) to be $0.19 M_{\odot}$, leading to a total mass of V833 Tau Aab of $1.03 M_{\odot}$. The system has a wide companion (WD0433+270) at 126 arcsec. The primary appears to be a member of Hyades group. However, the WD cooling age is not compatible with the Hyades age unless the rather exotic scenario of a Fe-core WD favoured by Catalán et al. (2008). Following Catalán et al. (2008) we then adopt the Hyades age (625 Myr) but a much older age (about 4 Gyr) cannot be ruled out. The high metallicity is compatible with Hyades membership.

26. GJ 3305 Member of $\beta$ Pic MG. Close visual binary discovered by K07. An orbital solution was derived by Delorme et al. (2012). The pair has also a wide companion (sep. 66 arcsec, see Feigelson et al. 2006), the F0V star 51 Eri.

27. HIP $21965=H D 30051$ Astrometric binary, with orbital solution derived by Goldin \& Makarov (2007). The star is a member of Tuc-Hor association.

28. DQ Tau SB2 with nearly identical components, member of Taurus star forming region. Orbital parameters from Mathieu et al. (1997) and primary mass from Daemgen et al. (2015)

29. $H I P 23296=H D 32115$ This is a slow rotating A type star without abundance anomalies. It is a short-period single-lined SB with orbital parameters derived in Fekel et al. (2006). The minimum mass is of $0.29 M_{\odot}$ (for a primary stellar mass of $\left.1.5 M_{\odot}\right)$. A very low mass star in wide orbit has been identified by De Rosa et al. (2014). V12 adopt an age of $125 \mathrm{Myr}$ from the position on CMD similar to Pleiades stars.

30. HIP $23418=$ GJ $3322=2$ MASS J05015881+0958587 Tight triple system, formed by a $12 \mathrm{~d}$ spectroscopic binary and an outer visual companion at 1.37 arcsec that strongly limits the region allowed for stable circumbinary planets around the central pair. We adopt the trigonometric distance from Riedel et al. (2014), the age from membership to $\beta$ Pic MG and masses from Tokovinin (2008).

31. L449-1AB See Bowler et al. (2015).

32. HIP $25486=H D 35850=A F$ Lep See Thalmann et al. (2014).

33. $A B$ Dor $A C=H I P 25647 A C=H D 37065 A C$ First of the two close pairs in the AB Dor quadruple system. Resolved by Close et al. (2005). Astrometric orbit has been derived by Guirado et al. (2006). We adopt these parameters in our analysis. The secondary $\mathrm{AB}$ Dor $\mathrm{C}$ is a very low mass star $\left(0.09 M_{\odot}\right)$. Included in the B07 survey.

34. $A B$ Dor $B a B b=H I P 25647 B a B b=H D 37065 B a B b$ Second pair in the $A B$ Dor quadruple system. Resolved into a 0.06 arcsec binary by Janson et al. (2007), included in the CH10 survey.

35. 2 MASS J05320450-0305291 = V1311 Ori $=$ TYC 4770-797-1 Close visual binary, member of $\beta$ Pic MG. Individual masses from Janson et al. (2012) and distance from L15.

36. HIP $30920 A=$ GJ 234 A = V575 Mon Spectroscopic, astrometric and visual binary. Parameters from Ségransan et al. (2000). The stellar age is uncertain but likely moderately young, considering the large X-ray emission, significant rotation and young disk kinematics. We adopt $150 \mathrm{Myr}$.

37. HIP $32104=H D 48097=26$ Gem $=H R 2466$ Member of Columba association according to Zuckerman et al. (2011) and Malo et al. (2013). Spectroscopic (Galland et al. 2005) and astrometric (HIPPARCos orbital solution) binary. Combining the spectroscopic solution with the inclination from HIPPARCos results in a companion mass of $0.51 M_{\odot}$ at $1.87 \mathrm{au}$. The secondary is most likely responsible for the $\mathrm{X}$-ray emission from the system.

38. HIP 35564 See Desidera et al. (2015).

39. HIP $36349=$ V372 Pup $=2$ MASS J07285137-3014490 $=G J$ 2060 Close visual system member of the AB Dor MG.

40. HIP 36414 See Desidera et al. (2015).

41. GJ $278 C=Y Y$ Gem $=$ Castor $C$ Eclipsing binary with similar components $(P=0.81 \mathrm{~d}, M=0.5975+0.6009$, Torres \& Ribas 2002). The other components of the Castor system (two SB with A type primaries) are at $72 \operatorname{arcsec}=$ 1070 au (total mass $4.83 M_{\odot}$, Torres \& Ribas 2002). Distance to the system from Torres \& Ribas (2002), based on reanalysis of HIPPARCCOs data. Member of Castor MG (Ribas 2003).

42. HIP $38160=H D 64185$ A close visual companion at 0.141 $\operatorname{arcsec}=4.8$ au has been reported by R13. This companion might also be responsible of the astrometric signature in Makarov \& Kaplan (2005). We adopt the mass of the companion from R13. The star listed in CCDM and WDS (CCDM J07492-6017B) at a projected separation of 23 arcsec is not physically associated. The star is a member of Carina-Near MG according to Zuckerman et al. (2006). 
43. HIP $39896 A=$ FP $C n c=$ GJ $1108 A$ The star is a probable member of Columba association according to B13. They also discovered a close visual companion (sep 0.25 arcsec).

44. HIP $39896 B=G J 1108 B$ Additional close pair (SB2) of $\mathrm{M}$ dwarf companions at a separation of 14 arcsec from HIP 39896 A. We adopt the discovery parameters by Shkolnik et al. (2012). Both pairs have been observed in deep imaging. There is a limited space of dynamical stability (from 23 to $68 \mathrm{au}$ ) for planets around the central binary, due to moderately wide orbit of the central binary and the presence of the outer pair.

45. $E M C h a=$ RECX7 See Thalmann et al. (2014).

46. $R S$ Cha $=$ HIP $42794=R E C X 8$ SB2 and EB with similar components, member of $\eta$ Cha open cluster. See Alecian et al. (2005) and references therein for a detailed description of the system. One of the components is also a pulsating $\delta$ Scu star.

47. $E Q C h a=R E C X 12$ Close visual binary member of $\eta$ Cha open cluster (B06). Flux ratio close to unity.

48. TYC 8927-3620-1 See Desidera et al. (2015).

49. HIP $45336=\theta$ Hya $=H D 79469$ The B9.5 star $\theta$ Hya was shown to have a WD companion with temperature $25000-31000 \mathrm{~K}$ from the analysis of the UV spectrum of the system (Burleigh \& Barstow 1999). Vennes et al. (1998) detected low amplitude RV variations and astrometric acceleration was detected from HIPPARcos data and from the difference of HIPPARCOS and historical proper motion (Makarov \& Kaplan 2005). Therefore, the period is expected to be or the order of a decade, but no orbital solution is available in the literature. We adopt the stellar masses from Holberg et al. (2013).

50. 1RXSJ091744.5+461229AB See Bowler et al. (2015). Individual masses from Janson et al. (2012).

51. HIP $47133=$ PYC J09362+3731 = GJ 9303 Short-period SB2, see Bowler et al. (2015) for details and references. As for other suspected tidally locked binaries we adopt an age of 4 Gyr.

52. HIP $49669=$ Regulus $=\alpha$ Leo $=H D 87901$ The presence of a spectroscopic companion was identified by Gies et al. (2008), with indication that the companion is a white dwarf. If this is the case, significant interaction between the components were expected to have happened, possibly explaining the extreme rotation of the (current) primary. Rappaport et al. (2009) modelled the evolution of the system, finding as the most likely initial configuration two stars of 2.3 and $1.7 M_{\odot}$ in short period (1-15 days). The current companion to the $3.4 M_{\odot}$ component is expected to be a $0.30 M_{\odot} \mathrm{He}$ WD. This scenario requires an age of the system older than 900 Myr.

The system is quadruple, as there is a close pair of low mass stars $(\mathrm{K} 2 \mathrm{~V}+\mathrm{M} 4 \mathrm{~V})$ at a projected separation of 175 arc$\mathrm{sec}=4000 \mathrm{au}$, whose physical association has been recently confirmed by Tokovinin et al. (2015). Therefore, we rely on the age indicators of the late-type component. The lack of lithium (Pallavicini et al. 1992a) indicate an age older than 500 Myr while the chromospheric and coronal emission yield an age slightly younger than the Hyades. We adopt an age of 600 Myr. This estimate indicates that some adjustments are needed in the description of the evolution of the system by Rappaport et al. (2009), which is not unexpected considering the theoretical uncertainties in the common envelope evolution.

53. $H I P 49809=H D 88215=H R 3991$ This is a rapidly rotating early F star and single-lined SB.
The minimum mass of the companion is $0.20 M_{\odot}$. Stellar age is obtained through isochrone fitting. Kinematics is compatible with young disk without association to any known group. The star hosts a debris disk.

54. HIP $50156=$ DK Leo $=$ GJ 2079 The star was suspected to have RV variations in the literature but without conclusive evidence of binarity. The star is also a $\Delta \mu$ binary. We retrieved 7 spectra from SOPHIE archive, which show RV variations of about $18 \mathrm{~km} \mathrm{~s}^{-1}$ (peak-to-valley) over about 1 month. The CCF indicates a single-lined SB. From the small variations of RVs taken in consecutive nights (which is also consistent with López-Santiago et al. 2010 measurements), it results that the period is likely of the order of months. Therefore, the large activity and fast rotation cannot explained by tidal locking but is rather due to youth. The star was classified as a member of Columba MG and $\beta$ Pic MG according to Brandt et al. (2014a) and Schlieder et al. (2012), Malo et al. (2013), respectively. However, the unknown system velocity represents a major source of uncertainty in these evaluations. Independently on the kinematics, we estimate as age of $150 \mathrm{Myr}$, taking the lithium non-detection (López-Santiago et al. 2010) into account. We also adopt as tentative binary parameters to estimate the limits of dynamical stability a period of $100 \mathrm{~d}$ and RV semiaplitude of $10 \mathrm{~km} \mathrm{~s}^{-1}$.

55. TWA 22 Originally proposed as TWA member, there are no adequate kinematic data according to Torres et al. (2008). Teixeira et al. (2009) derived system parallax, proposing association with the $\beta$ Pic MG, that we adopt here. Orbit from Bonnefoy et al. (2009).

56. TYC 7188-0575-1 See Desidera et al. (2015).

57. CHXR 74 Binary and stellar parameters from Joergens et al. (2012).

58. TWA $5 A a b$ The central pair was first resolved by Macintosh et al. (2001) and its orbit was derived by Konopacky et al. (2007) and recently refined by Köhler et al. (2013), obtaining a period of $6.025 \mathrm{yr}$, a semimajor axis of 63.7 mas and an eccentricity of 0.755 . Adopting the recently derived trigonometric parallax (Weinberger et al. 2013), the sum of the masses of the components is $0.90 M_{\odot}$ and the semimajor axis 3.2 au. Torres et al. (2003) identified TWA5 as a very short period single-lined SB, with period 1.37 days and RV semiamplitude $20 \mathrm{~km} \mathrm{~s}^{-1}$. Therefore the system should include three stellar components, but some concerns on on the existence of the short-period companion were presented by Weinberger et al. (2013). An additional companion of sub-stellar mass (TWA5 B) to the pair was discovered by Lowrance et al. (1999), Webb et al. (1999) at a projected separation of $1.95^{\prime \prime}=97.7$ au from TWA5Aab. The mass of TWA5B is of $20 M_{\mathrm{J}}$ according to Lowrance et al. (1999) and Webb et al. (1999) and $25 M_{\mathrm{J}}$ according to Neuhäuser et al. (2010), Chauvin et al. (2010). A preliminary orbital solution indicates a semimajor axis of 127 au with eccentricity of 0.24 (Köhler et al. 2013).

59. HD 102982 Very active star, probable SB2 according to Soderblom et al. (1998). A FEROS spectrum from ESO archive confirms the SB2 nature of the system. Kiraga (2012) classified the star as a contact eclipsing binary with period of $0.277 \mathrm{~d}$. Nordström et al. (2004) gives RV $=-67.3 \pm$ $4.6 \mathrm{~km} \mathrm{~s}^{-1}$ (1 measurement), which would imply kinematic parameters typical of an old star. However, the binarity may have significant impact on the RV. In any case, there is a good chance that the large activity is due to tidally-enhanced rotation and not to young age. We then adopt an age of $4 \mathrm{Gyr}$. 
L05 identified an additional companion at $0.9^{\prime \prime}$ (spectral type M5V), making the system triple.

60. TWA 23 Member of TW Hya association. RV variability was discovered by Bailey et al. (2012). Their 14 measurements does not allow a unique orbital solution; they list three equally good orbits. Conservatively, we derive the limit for dynamical stability adopting their solution with the longest period. We adopt the trigonometric parallax and stellar mass from Weinberger et al. (2013).

61. HIP $59960=H D 106906$ Member of LCC, the star was shown to host a $11 M_{\mathrm{J}}$ companion at a projected separation of 650 au (Bailey et al. 2014). Images from JL13 were used in the discovery paper. Very recently, Lagrange et al. (2016), showed that the central star is an SB2 system. The star has also a significant infrared excess, indicating the presence of a massive debris disk, which have been recently spatially resolved with SPHERE and GPI (Lagrange et al. 2016; Kalas et al. 2015).

62. G 13-33 Young M dwarf resolved into a tight binary by B15. The system is not associated with known moving groups. B15 adopt an age between 10 to 300 Myr from Shkolnik et al. (in prep.) We adopt $150 \mathrm{Myr}$.

63. HIP 60553 Identified as SB2 in Torres et al. (2006), with an estimated magnitude difference of $0.5 \mathrm{mag}$ in V. The star is also flagged as stochastic solution in the original HIPPARCOS catalog. Orbital solution is not known. Therefore, we are not able to determine whether the very large coronal emission $\left(\log L_{\mathrm{X}} / L_{\text {bol }}=-2.93\right)$ and fast rotation (period 0.89 days, Koen \& Eyer (2002)) are due to youth or tidal locking. From the lack of lithium (Torres et al. 2006), a lower limit of $400 \mathrm{Myr}$ on stellar age is derived. The space velocities derived using the single-epoch RV from Torres et al. (2006) are far from locus typical of young stars, so we argue it is a old star tidally locked by a close companion. We adopt and age of 4 Gyr.

64. GJ 3729 Young M dwarf resolved into a tight binary by B15. The system is a possible member of Tuc-Hor MG (Shkolnik et al. 2012).

65. TWA 20 Young star classified as SB2 by Jayawardhana et al. (2006) and Elliott et al. (2014). The large RV difference between the components (at least $125 \mathrm{~km} \mathrm{~s}^{-1}$ ) indicate a rather short orbital period. We adopt the trigonometric parallax by Weinberger et al. (2013). Elliott et al. (2014) reject membership on TWA on the basis of their revised system RV. The lithium content (da Silva et al. 2009) indicates an age intermediate between TWA and beta Pic MGs.

66. HIP $62983=H D 112131$ Close stellar companion resolved by lunar occultation and speckle interferometry (Africano et al. 1975; Mason 1996). The CHARM2 catalogue (Richichi et al. 2005) quote a projected separation of 0.320 arcsec and brightness ratio of 5.2 in $V$ band. From this, we infer that the secondary is a late F star. V12 quote a separation of 0.04 arcsec. We adopt the projected separation from occultation for the derivation of the critical semimajor axis for dynamical stability. We adopt the age of $125 \mathrm{Myr}$ from V12.

67. HIP $63742=H D 113449=P X$ Vir Member of $\mathrm{AB}$ Dor MG according to Zuckerman et al. (2004) and Torres et al. (2008). Close companion detected by HIPPARCos astrometry (with orbital solution), radial velocity (Griffin 2010) and direct imaging (Evans et al. 2012) The orbital solution by Evans et al. (2012) is adopted here.

68. HIP $63962=H D 113706 \mathrm{G} 0$ star classified as member of LCC. J13b noted the elongated PSF, indicating an unresolved binary with projected separation well below 50 mas. The binarity is further supported by the difference among the two RV measurements available in the literature (12.6 \pm $0.2 \mathrm{~km} \mathrm{~s}^{-1}$ and $4.8 \pm 1.3 \mathrm{~km} \mathrm{~s}^{-1}$ from Chen et al. 2011 and Bobylev et al. 2007 respectively). We tentatively adopt a projected separation of 30 mas and a mass ratio close to unity.

69. 2MASS J13215631-1052098 Close visual binary (L15), probable member of TWA following Riaz et al. (2006).

70. HIP $66001=H D 117524 \mathrm{G} 8$ star classified as member of LCC. J13b noted the elongated PSF, indicating an unresolved binary with projected separation below 50 mas. The binarity is further supported by the astrometric acceleration detected by HIPPARCOS and the difference among the two $\mathrm{RV}$ measurements available in the literature $\left(7.4 \mathrm{~km} \mathrm{~s}^{-1}\right.$ and $2.7 \pm 1.2 \mathrm{~km} \mathrm{~s}^{-1}$ from SACY and Bobylev et al. (2007) respectively). We tentatively adopt a projected separation of 30 mas and a mass ratio close to unity.

71. HIP $72399=H D$ 130260A See Desidera et al. (2015).

72. $H I P 74045=H D 135363=I U$ Dra Close companion detected by B07 and GDPS $(\Delta H=4.0)$. Montes et al. (2001a) classified the star as a member of the IC2391 MG. Makarov et al. (2007) support this association by noting a possible close encounter with IC 239124 Myr ago with small relative velocity. Bubar et al. (2007) estimated an age of $35_{-6}^{+14} \mathrm{Myr}$ from isochrone fitting, further supporting the association. The activity indicators and lithium abundance suggest an age similar or younger than the Pleiades. We adopt an age of $50 \mathrm{Myr}$.

73. HIP $76267=\alpha C r B=H D 139006$ Double-lined spectroscopic and eclipsing binary composed by a B9.5 primary and $\mathrm{G}$ secondary. We adopt the individual masses and orbit from Tomkin \& Popper (1986). The space velocities are compatible with UMa membership, as previously proposed by King et al. (2003). The X-ray luminosity is comparable to Hyades star, if one assumes it is originating from the solartype secondary. We then adopt an age of 500 Myr. A circumbinary debris disk was resolved by Booth et al. (2013) on the basis of Herschel data.

74. IRXS J153557.0-232417 = GSC 06764-01305 Close visual binary discovered by Kraus et al. (2008). Masses of the components from Kraus et al. (2008).

75. HIP $76629=$ HD $139084=$ V343 Nor See Thalmann et al. (2014).

76. HIP 77858 SB1, orbit from Levato et al. (1987).

77. HIP 78104 SB1, orbit from Levato et al. (1987).

78. $R X$ J155734.4-232112 = V1148 Sco $=$ ScoPMS 17 The star was resolved as close visual binary in Kraus et al. (2008) and L14, with some discrepancy in the mass ratio between the two sources. We adopt the individual masses by Kraus et al. (2008).

79. HIP 78168 SB1, orbit from Levato et al. (1987).

80. HIP 78196 A very low mass star at small separation was discovered by Hinkley et al. (2015) using the sparse aperture masking technique.

81. HIP 78207 SB2 discovered by Dahm et al. (2012). Only single-epoch RV difference between the components available.

82. HIP $78265=H D 143018$ Double-lined spectroscopic and eclipsing binary. Orbital parameters from Stickland et al. (1996).

83. IRXS J160210.1-2241.28 = V1154 Sco Short-period spectroscopic binary discovered by Mathieu et al. (1989). An additional system of lines at constant RV is also reported. A visual companion was discovered at about 0.30 arcsec making the system triple (Ghez et al. 1993; Köhler et al. 2000). 
The visual component is likely the responsible for the additional spectral signature. We adopt the stellar masses of the visual components by Kraus et al. (2008) and the minimum mass from the spectroscopic orbit for the unseen spectroscopic component. The system configuration leaves little dynamical room in our planet-search zone, as the critical semimajor axis due to the wide component is at about $12 \mathrm{au}$, corresponding to just 0.09 arcsec at the distance of Upper Scorpius region, while the limit for stability of planet around the whole triple system is too wide for being considered in this work (193 au).

84. [PGZ2001]J160341.8-200557 SB2 discovered by Dahm et al. (2012). Only single-epoch RV difference between the components available.

85. IRXJ 160355.8-203138 Close visual binary in Upper Scorpius.

86. $1 R X S$ J160446.5-193031 $=$ V1156 Sco $=$ ScoPMSO27 Close visual binary discovered by Kraus et al. (2008). Masses of the components from Kraus et al. (2008).

87. [PGZ2001]J160545.4-202308 SB2 discovered by Dahm et al. (2012). Only single-epoch RV difference between the components available. Dahm et al. (2012) also reported a visual companion candidate identified on the HIRES guide camera images, but separation and magnitude difference are not listed.

88. HIP $78977=H D 144548=E P I C-204506777$ This is a triple eclipsing system member of Upper Scorpius association. A close eclipsing system was originally identified by Kiraga (2012). Alonso et al. (2015) revised the period of the shortperiod eclipsing binary and identified additional eclipses with a period of 33 days, thanks to the Kepler- 2 photometric time series. We adopt the system parameters from this latter study. The direct imaging observations allow to probe the presence of sub-stellar companions around the three components of this tight triple system. The system was also reported to have IR excess at $24 \mu \mathrm{m}$ (Chen et al. 2011).

89. 1RXS J160814.2-190845 = TYC 6209-735-1 = GSC 0620900735 Spectroscopic binary discovered by Guenther et al. (2007). The companion has also been resolved by Kraus et al. (2008) from sparse aperture mask observations at a projected separation 25 mas $=3.6$ au. The mass of the secondary estimated by Kraus et al. (2008) is similar to the minimum mass derived by the RV orbital solution.

90. HIP $79097=H D 144823 \mathrm{~J} 13 \mathrm{~b}$ noted the elongated PSF, indicating an unresolved binary with projected separation below 50 mas. At odds to HIP 63962 and HIP 66001, which were also proposed as binaries by J13b due to PSF elongation, there are no multiple RV measurements available in the literature to confirm the binarity. We tentatively adopt a projected separation of 30 mas and a mass ratio close to unity. The star has an additional component at 0.8 arcsec, making the system a likely triple (J13b).

91. HIP 79404 SB1, orbit from Levato et al. (1987). Member of US.

92. $1 R X S$ J161318.0-221251 = TYC 6213-0306-1 = BD-21 4301 SB2 with nearly identical components discovered by Guenther et al. (2007). These authors also derived the orbital solution. We adopt the primary mass by L14.

93. HIP 79643 B Triple system, formed by a F2 star, separated by 1.24 arcsec from a close pair (projected separation 47 mas, see L14) which is the target considered in our study. Masses from L14. Member of US

94. HIP $81266=\tau$ Sco $=H D 149438=H R 6165$ This early B star, member of US, was recently resolved in a close binary (projected separation $21.52 \pm 0.27$ mas) by interferometric observations (Rizzuto et al. 2013).

95. HIP $84586=H D 155555$ Triple system, member of $\beta$ Pic MG. The spectroscopic binary with a period of 1.68 days and a mass-ratio close to unity $\left(M_{A}=1.06 M_{\odot}\right.$ and $M_{C}=$ $\left.0.98 M_{\odot}\right)$ has a distant $\left(\rho=33^{\prime \prime}\right)$ companion with $M_{B}=$ $0.25 M_{\odot}$.

96. HIP $84642=H D 155915=$ V857 Ara Close binary star, possible member of Tuc-Hor association according to Zuckerman et al. (2011) and further confirmed as member by Malo et al. (2013). The age indicators are fully consistent with the membership assignment.

97. HIP $86346=H D 160934$ Member of AB Dor MG. A close companion was identified by both RV and direct imaging (Gálvez et al. 2006; Lafrenière et al. 2007; Hormuth et al. 2007; Griffin \& Filiz Ak 2010; Evans et al. 2012). The composite orbital solution by Evans et al. (2012) was adopted. Weis (1991) reported a companion at 20", confirmed by 2MASS observations (see Lowrance et al. 2005).

98. HIP $88848=H D 166181=V 815$ Her Triple system. This short period spectroscopic binary ( $p=1.8$ days) has been found by Fekel et al. (2005) to have a further companion with $p=5.7 \mathrm{yr}$ on a quite eccentric orbit $(e=0.76)$. Fekel et al. (2005) also reports a mass of $0.37 M_{\odot}$ and $0.79 M_{\odot}$ for the close and the distant companion respectively. An astrometric solution is also reported, with $a=$ $4.1 \mathrm{au}=0.13^{\prime \prime}$. The outer companion was resolved in GDPS. As the very high coronal activity should be induced by the close companion, we do not use the X-ray luminosity for the age determination. The lithium EW suggests an age of $125 \mathrm{Myr}$ (to be taken with caution because of the blending of three objects, dedicated modelling would be needed).

99. $C D-641208 A=T Y C$ 9077-2489-1 Close visual binary resolved by Biller et al. (2007), Chauvin et al. (2010) at a projected separation of about 0.17 arcsec and with $\Delta K=$ 2.3 mag. The pair has a wide companion, the A7V star HIP $92024=$ HD $172555=$ HR 7012 at $70 \operatorname{arcsec}=2000 \mathrm{au}$ projected separation, from which we took the trigonometric parallax of the system. The system is a member of $\beta$ Pic MG. 00. HIP $92919=$ HD $175742=$ V775 Her Single-lined SB (period 2.879 days, circular orbit). Plavchan et al. (2009) discovered $24 \mu \mathrm{m}$ excess. The star is a BY Dra variable, with photometric period similar to the orbital one, indicating tidal locking. This is likely responsible for the enhanced activity level of the star. The kinematic parameters $(U, V, W=24.5$, $0.0,-22.6 \mathrm{~km} \mathrm{~s}^{-1}$, using center of mass velocity from SB9 orbit) put the system far from the region of very young stars and close to UMa group. Membership to UMa is assigned by Montes et al. (2001b) and considered possible by King et al. (2003) J13 adopted an age of 40-60 Myr from Plavchan et al. (2009). Marginal detection of lithium have been reported by Strassmeier et al. (2000) and White et al. (2007) while only upper limits by Mishenina et al. (2012). These values are compatible with a star of the age of UMa. We then adopt 500 Myr.

101. HIP $94050=H D 177996$ The star is a short-period SB2 (Soderblom et al. 1998), but the orbital solution is not available. The line depth ratio is about 0.5 at $6700 \AA$. Lithium was detected, with an EW likely larger than Hyades of similar color, indicating a true moderately young star rather than a tidally-locked system. Adopting a RV of -38.4 from Soderblom et al. (1998), kinematic parameters similar to the Hyades are derived $(U V W=-40.5,-14.2,5.3)$. We adopt an age of $400 \pm 200 \mathrm{Myr}$. 
102. HIP $94863=$ HD 180445 The star is a short period SB2 108. TYC 5206-0915-1 See Desidera et al. (2015). (Cutispoto et al. 2002, G8V+K5V). A preliminary orbital 109. HIP $105404=H D 202917=$ BS Ind See Thalmann et al. solution was provided by Tokovinin et al. (2006). They also found evidence for a wide-separation tertiary component at 9.4 arcsec. We adopt the individual masses from MSC and we assume circular orbit due to the short period. No lithium was detected by Cutispoto et al. (2002), Soderblom et al. (1998), with limits corresponding to ages older than about 500 Myr. The rotation period by Kiraga (2012) is very close to the orbital period. Therefore the large coronal and chromospheric emission appear to be due to tidally induced rotation and not to young age. The main sequence status of both components and the thin disk kinematics put an upper limit of about $8 \mathrm{Gyr}$. We then adopt an age of $4 \mathrm{Gyr}$.

103. HIP $95149=H D 181321=G J 755$ Reported as a SB in Nordström et al. (2004) (scatter of RV of $2.3 \mathrm{~km} \mathrm{~s}^{-1}$ over about 9 years) and Guenther \& Esposito (2007) (trend of $-1.4 \mathrm{~km} \mathrm{~s}^{-1} / \mathrm{yr}$, with a possible curvature over $1.2 \mathrm{yr}$ ). The astrometric acceleration was also detected by HIPPARCos. We then argue that the companion is most likely a low mass star with a period of several years. A spectral type later than $\mathrm{K} 5$ is expected from the lack of signature of the secondary in the spectra (Cutispoto et al. 2002). The age indicators point to a moderately old age, compatible with membership in Castor MG proposed by Ribas (2003).

104. HIP $97255=H D 186704$ This star shows RV variations of at least $5 \mathrm{~km} \mathrm{~s}^{-1}$ peak-to-valley (Nidever et al. 2002; Nordström et al. 2004; Tremko et al. 2010). Tokovinin (2014) quote a period of 3990d from a priv. comm. by D. Latham. As the minimum mass of the spectroscopic companion is not included in this study, we adopt $0.3 M_{\odot}$ for the calculation of the stability limits The star has a wide compan- 11 ion, the flare star V1406 Aql, at 9 arcsec. Zuckerman et al. (2013) classified the system as a probable member of the Octans-Near Association. The age indicators are compatible with an age similar to the Pleiades.

105. 2MASSJ19560294-3207186 Close visual pair with an additional component, the M0 star TYC 7443-1102-1, at 26 arcsec. This triple system is a probable member of $\beta$ Pic MG.

106. HIP $100751=H D 193924=\alpha$ Pav This star, member of Tucana association, is a close spectroscopic binary (SB9). The minimum mass of the companion is $0.26 M_{\odot}$.

107. HIP $101800=\iota \mathrm{Del}=H D 196544$ This star is a spectroscopic binary with an Am primary. The short-period orbit from SB $(P=11.039 \mathrm{~d} ; e=0.23)$ yields a minimum mass of $0.49 M_{\odot}$ for a primary stellar mass of $2.0 M_{\odot}$. The companion is then most likely a early $\mathrm{M}$ or a $\mathrm{K}$ dwarf. The star also shows IR excess (Rhee et al. 2007; Morales et al. 2011). This star was observed in deep imaging by R13 and B13. These studies provide discrepant age values. R13 assumed an age of $30 \mathrm{Myr}$ from Rhee et al. (2007) while N13 list their own determination of a median age of $272 \mathrm{Myr}$ (69-444 Myr 95\% limits). To further investigate the issue, we consider the kinematic of the system, adopting the center of mass velocity from SB9 and distance and proper motions from Van Leeuwen (2007). the space velocities results $U, V, W=-7.7,-4.2,-8.2$. These are quite far from those of the moving groups younger than $100 \mathrm{Myr}$ and compatible within error with those of the Castor MG (Ribas 2003). An additional indirect evidence against a very young age comes from the lack of detection of the system (whose X-ray emission should be dominated by the secondary, unless it is a WD) using ROSAT. Therefore, we adopt the age of the Castor MG as given in Ribas (2003) (320 Myr).

$$
\text { (2014). }
$$

110. HIP $105441=$ HD $202746=$ V390 Pav This star was classified as a new potential member of Tuc-Hor association in Zuckerman et al. (2001) but it was rejected by Song et al. (2003) because of its low lithium content. Membership to $\beta$ Pic MG is instead supported by Malo et al. (2013). Radial velocity is variable with peak-to-valley difference of at least $30 \mathrm{~km} \mathrm{~s}^{-1}$ (Nordström et al. 2004; Torres et al. 2006; Gray et al. 2006) and Gray et al. (2006) noted the possible presence of blending in the violet part of their optical spectrum. The star has a wide (26 arcsec projected separation) companion, TYC 9114-1267-1. Both components were observed by K07. The very similar proper motions and the fact that the photometric distance of TYC 9114-1267-1 is compatible with the trigonometric parallax of HIP 105441 suggest physical association, with the RV difference being due to binarity of the primary. However, TYC 91141267-1 (K7V) has detectable lithium $(E W=15 \mathrm{~mA})$ in SACY, suggesting an age of about 30-50 Myr, while HIP 105441 (K2V) has no detectable lithium (Song et al. 2003; Torres et al. 2006), corresponding to a lower limit on stellar age of about 400 Myr. HIP 105441 shows indication of enhanced activity (Arriagada 2011) and rotation (Kiraga 2012), which would indicate age of about $100 \mathrm{Myr}$, but considering the lack of lithium, we favour tidal locking as the source of these characteristics. We then adopt an age of $4 \mathrm{Gyr}$ but we note that further studies are needed for a characterisation of this object and to investigate its physical association with TYC 9114-1267-1.

111. HIP $107556=\delta$ Cap $=H D 207098=$ GJ 837 See Thalmann et al. (2014).

112. [FS2003] $1136=1 R X S$ J214906.4-641300 Resolved as a close visual binary by $\mathrm{CH} 10$. Stellar parameters from $\mathrm{CH} 10$.

113. HIP 108195 Triple system formed by a close pair of F stars and a M5-M7 companion at 4.9 arcsec, identified by Chauvin et al. (2010). The inner pair has a preliminary orbit in WDS. The system is a member of Tucana association.

114. HIP $109901=H D 211087=$ CS Gru See Thalmann et al. (2014).

115. GJ $860=$ HD 239960 B Close visual binary with orbital solution. Individual masses from Henry et al. (1999). Age from $\mathrm{H} 10$.

116. $P P M 366328=$ TYC 9129-1361-1 Classified as possible member of Tuc-Hor association (Zuckerman et al. 2001), Torres et al. (2006) showed that instead the star is an SB2 $(\Delta V=1 \mathrm{mag})$ with no detectable lithium, indicating an age older than the Hyades. The different RVs as measured by Zuckerman \& Webb (2000) and Torres et al. (2006) also support binary. The very fast rotation $\left(v \sin i=88 \mathrm{~km} \mathrm{~s}^{-1}\right)$ and bright X-ray emission $\left(\log L_{\mathrm{X}} / L_{\mathrm{bol}}=-3.38\right)$ are then likely due to tides of the companion. There is another companion at $24 \operatorname{arcsec}$ (Neuhäuser et al. 2003), classified as M2 by Riaz et al. (2006). Taking into account both components, we adopt a distance of $60 \mathrm{pc}$, and, assuming tidal locking as responsible of the enhanced activity of the SB2 system, an age of 4 Gyr. Masses of the components from mass-luminosity relations.

117. HIP $116003=$ GJ $1284=2$ MASSJ23301341-2023271 This star was classified as SB2 by Torres et al. (2006). The orbital solution is not available. It was classified as candidate member candidate of Columba association by Malo et al. (2014) We adopt the trigonometric distance by Riedel et al. (2014). 


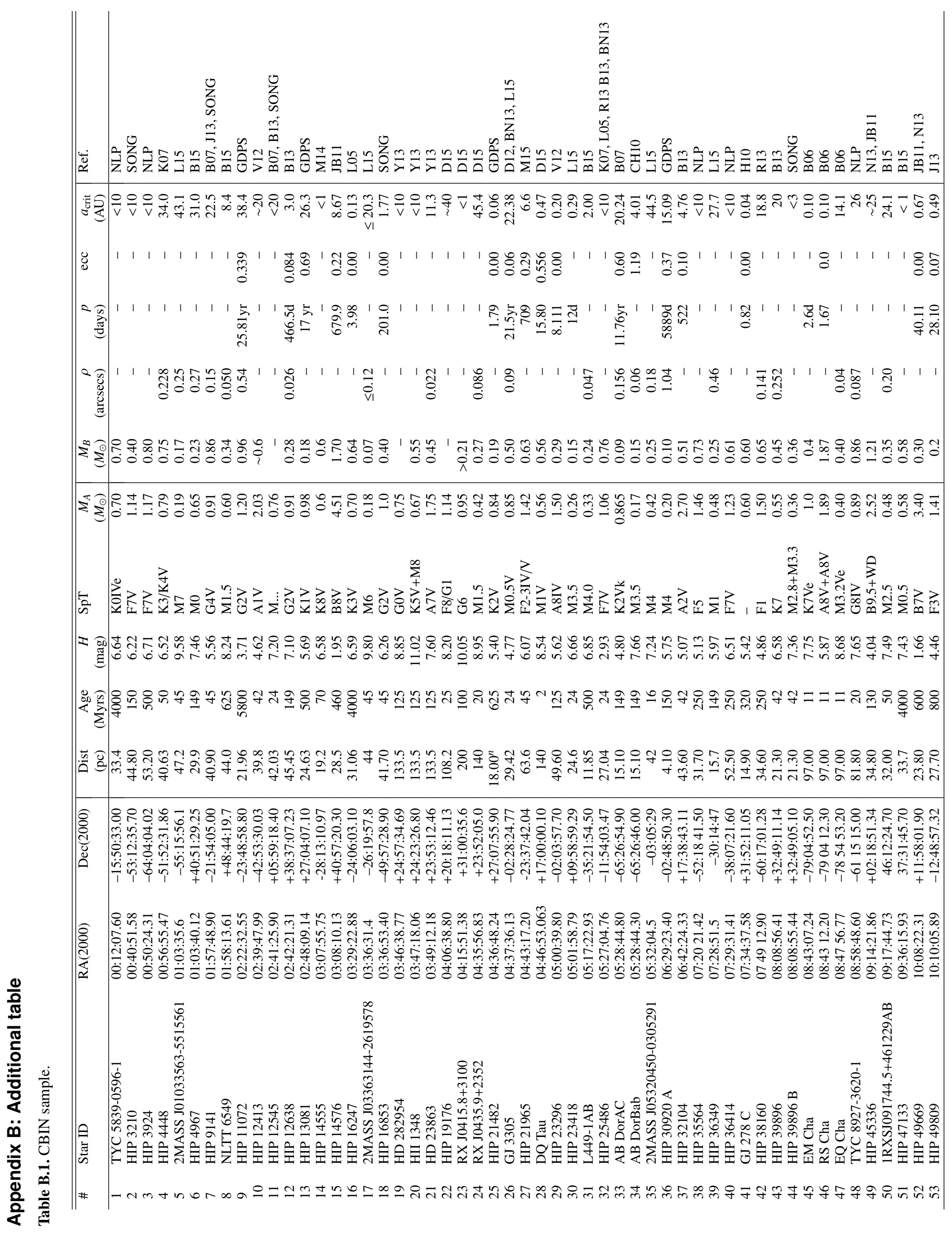




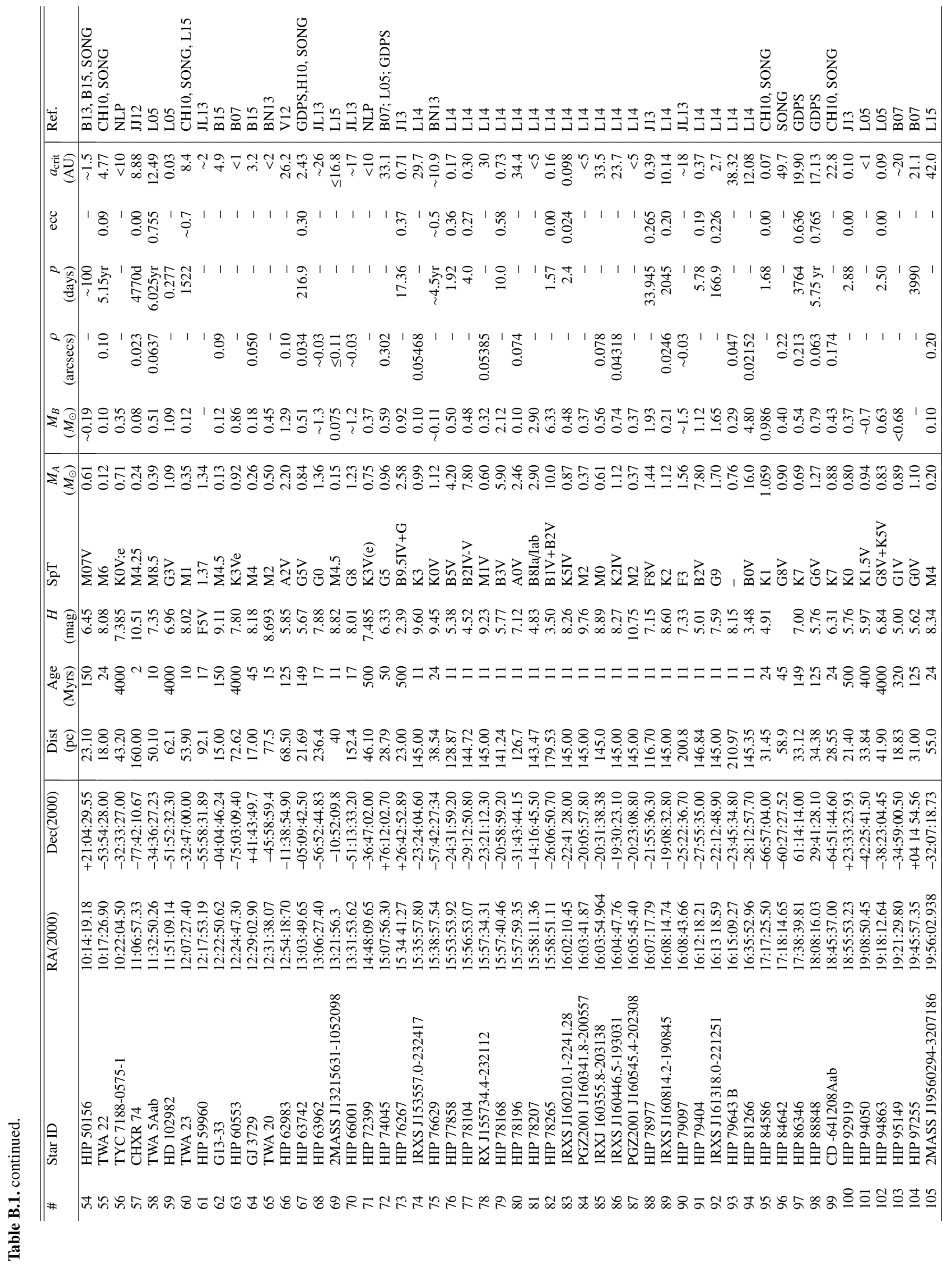


A\&A 593, A38 (2016)

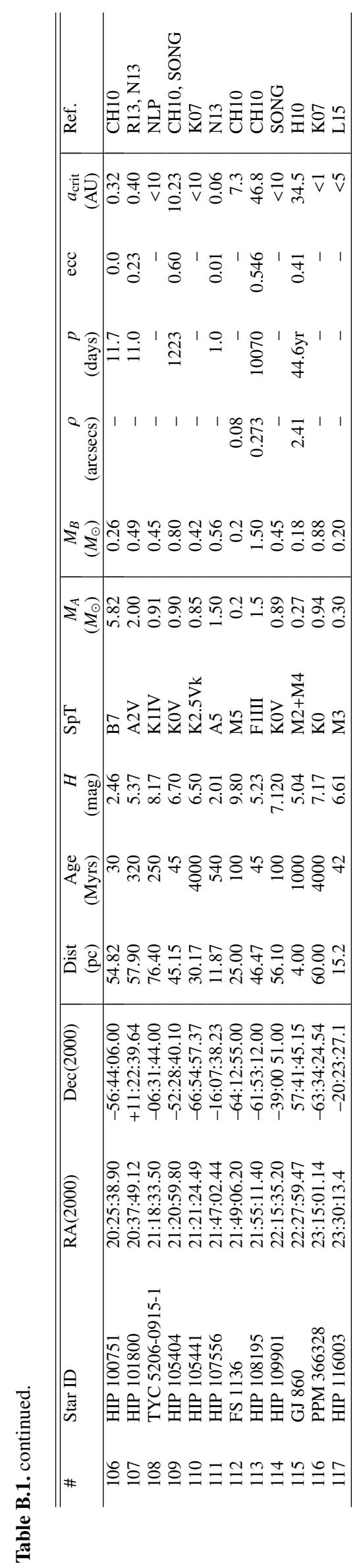

